\title{
Transversal Ordered Interval and Edges Spaces, Fixed Points and Applications
}

\author{
Milan R. TASKOViĆ
}

\begin{abstract}
In this paper we formulate a new structure of spaces which we call it transversal (upper, middle or lower) ordered interval spaces. Also, we formulate a new structure of spaces which we call it transversal (upper, middle or lower) ordered edges spaces. We introduce this concepts as a natural extension of transversal probabilistic, Fréchet's, Kurepa's and Menger's spaces. This are concepts of transversal spaces with nonnumerical transverses. Transversal ordered interval and edges spaces are new concepts of spaces in the fixed point theory and further a new way in nonlinear functional analysis. In this sense, we introduce notions of the ordered interval contractions on upper and lower transversal ordered interval spaces and prove some fixed point statements as and further applications. This concept have very important applications in numerical analysis and quantum particle physics by L. Collatz [Funktionalanalysis und Num. Math. Springer-Verlag, 1964].
\end{abstract}

\section{INTRODUCTION, FACTS AND HISTORY}

The notion of distance $d(x, y)$ between points $x$ and $y$ is very old and is essential connection with measurements. Certainly, the notion is present in works of Thales of Milet (circa 624B.C.-546B.C.), one of the seven wise men of the Antic Greece, the first man who predicted the eclipse of the sun (for the year 585 B.C.); he performed various calculations with distances and angles.

A perpetual monument to the old notion of distance is the Pythagora Theorem on triangles of Pythagoras of Samos (circa 560 B.C. - 480 B.C.). In fact, this theorem was already known in Babylon at the time of King Hammurabi (circa 1728 B.C. - 1686 B.C.).

2000 Mathematics Subject Classification. Primary: 54E15, 47H10, 05A15. Secondary: $54 \mathrm{E} 35,54 \mathrm{H} 25$.

Key words and phrases. General ecart, distance, Fréchet's spaces, Kurepa's spaces, Menger's spaces, transversal spaces, nonnumerical transverses, transversal probabilistic spaces, transversal ordered interval spaces, transverse, bisection functions, fixed points, ordered interval contractions, intuitionistic fuzzy metric spaces, $\mathcal{L}$-fuzzy metric spaces, edges spaces, middle transversal interval space, middle transversal edges space, Tasković's transversal spaces. 
Presumably, however, it was a mathematician of the Pythagorean school who first proved the Pythagorean theorem. This theorem appears as Proposition 47 in Book I of Euclid's Elements (300 B.C.).

The concept of an abstract metric space, introduced by M. Fréchet in 1905, furnishes the common idealization of a large number of mathematical, physical and other scientific constructs in which the notion of a distance appears.

The objects under consideration may be most varied. The may be points, functions, sets, and even the subjective experiences of sensations. A generalization which was first introduced by K. Menger in 1942 and, following him, is called a statistical metric space.

In 1934 Dj. K u r e p a defined pseudodistancional spaces, with the nonnumerical distance, which play an important role in nonlinear numerical analysis (see: L. Collatz [11]). After that several authors investigated the distance functions taking values in partially ordered sets (A. A p p e r t, M. Fr é c h e t, J. C o l m e z, R. Doss, Ky Fan, Papić, Frink and others in the year's 40's and 50's). See also: A z zimond i-S c a r a velli [6], Frink [23], and Wald [60].

Concept of transversal spaces where introduced in 1998 by Tasković [54] as a nature extension of Fréchet's, Kurepa's, and Menger's spaces in the following sense. Also see: Tasković [51-58].

Let $X$ be a nonempty set and let $P:=(P, \preccurlyeq)$ be a partially ordered set. The function $\rho: X \times X \rightarrow P$ is called an upper transverse on $X$ (or upper transversal) iff: $\rho[x, y]=\rho[y, x]$, and if there is an upper bisection function $g: P \times P \rightarrow P$ such that

$$
\rho[x, y] \preccurlyeq \sup \{\rho[x, z], \rho[z, y], g(\rho[x, z], \rho[z, y])\}
$$

for all $x, y, z \in X$. A transversal upper space is a set $X$ together with a given upper transverse on $X$. The element $\theta=\rho[x, y] \in P$ if and only if $x=y$ is called spring of space $X$.

Let $k=\aleph_{\alpha}(\alpha \geq 0)$ be a regular cardinal. Call a topological space $X$ an upper $k$-transversal space or a $g\left(D_{\alpha}\right)$-space if there exists $\rho: X \times X \rightarrow$ $\omega_{\alpha} \cup\left\{\omega_{\alpha}\right\}:=W$ such that $\rho[x, y]=\omega_{\alpha}$ if and only if $x=y, \rho[x, y]=\rho[y, x]$, and if there is $g: W \times W \rightarrow W$ such that (A) for all $x, y, z \in X$.

We notice, Fréchet's spaces are important examples of upper $k$-transversal spaces.

Open problem 1. Does for every regular cardinal $k \geq \aleph_{0}$ there exists an upper $k$-transversal (i.e., an $g\left(D_{\alpha}\right)$-space) nonlinearly orderable topological space? Does some of upper transversal spaces have the fixed point property?

Let $X$ be a nonempty set and we chosen an upper bisection function $g:\left(\mathbb{R}_{+}^{0}\right) \rightarrow\left(\mathbb{R}_{+}^{0}\right):=[0,+\infty)$ defined by

$$
g(s, t)=\psi(s)+\tau t \quad\left(\tau \geq 1, \psi: \mathbb{R}_{+}^{0} \rightarrow \mathbb{R}_{+}^{0}\right)
$$

for a self-map $\psi$ with the property $\psi(x) \rightarrow 0(x \rightarrow 0)$, then $X$ is an example of transversal upper space, which where introduced in 1974 by M. Cicchese [9]. Special case of this spaces recently is considered Czerwik [13]. 
In connection with the preceding, let $P:=(P, \preccurlyeq)$ be a partially ordered set. The function $\rho: X \times X \rightarrow P$ is called a lower transverse on $X$ (or lower transversal) iff: $\rho[x, y]=\rho[y, x]$ and if there is a lower bisection function $d: P \times P \rightarrow P$ such that

$$
\inf \{\rho[x, z], \rho[z, y], d(\rho[x, z], \rho[z, y])\} \preccurlyeq \rho[x, y]
$$

for all $x, y, z \in X$. A lower transversal space is a set $X$ together with a given lower transverse on $X$. The element $\theta=\rho[x, y] \in P$ if and only if $x=y$ is called spring of space $X$.

Middle transversal spaces. As a new space (or as third side of a given space) is a middle transversal space by Tasković [54]. In this sense, a middle transversal space (or middle space) is an upper transversal space and a lower transversal space simultaneus. For further facts of the middle transversal spaces see: Tasković [54] and [56].

In the theory of metric spaces, as and in the theory of transversal spaces, it is extremely convenient to use a geometrical language inspired by classical geometry.

Thus elements of a transversal space will usually be called points. Given an upper transversal space $(X, \rho)$, with the upper bisection function $g: P \times P \rightarrow P$ and a point $\alpha \in X$, the open ball of center $\alpha$ and radius $r \in P$ is the set

$$
g(B(\alpha, r))=\{x \in X \quad: \quad \rho[\alpha, x] \preccurlyeq r\},
$$

till for given a lower transversal space $(X, \rho)$, with the lower bisection function $d: P \times P \rightarrow P$ and a point $\alpha \in X$, the open ball of center $\alpha$ and radius $r \in P$ is the set

$$
d(B(\alpha, r))=\{x \in X \quad: \quad r \preccurlyeq \rho[\alpha, x]\} .
$$

In connection with this, for any nonempty set $Y$ in the upper transversal space $(X, \rho)$, the diameter of $Y$ is defined as

$$
\operatorname{diam}(Y):=\sup \{\rho[x, y] \quad: \quad x, y \in Y\} ;
$$

where $A \subset B$ implies $\operatorname{diam}(A) \preccurlyeq \operatorname{diam}(B)$. On the other hand, for any nonempty set $Y$ in the lower transversal space $(X, \rho)$, the diameter of $Y$ is defined as

$$
\operatorname{diam}(Y):=\inf \{\rho[x, y] \quad: \quad x, y \in Y\} ;
$$

where $A \subset B$ implies $\operatorname{diam}(B) \preccurlyeq \operatorname{diam}(A)$. We notice that this notations are essential for further work.

Let $k=\aleph_{\alpha}(\alpha \geq 0)$ be a regular cardinal. Call a topological space $X$ a lower $k$-transversal space or $d\left(D_{\alpha}\right)$-space if there exists the function $\rho: X \times X \rightarrow$ $\omega_{\alpha} \cup\left\{\omega_{\alpha}\right\}:=W$ such that: $\rho[x, y]=\omega_{\alpha}$ if and only if $x=y, \rho[x, y]=\rho[y, x]$, and if there is $d: W \times W \rightarrow W$ such that (B) for all $x, y, z \in X$.

Open problem 2. Does for every regular cardinal $k \geq \aleph_{0}$ there exists a lower $k$-transversal (i.e., an $d\left(D_{\alpha}\right)$-space) nonlinearly operable topological space? Does some of lower transversal spaces have the fixed point property?

We notice, in connection with this problem, that work of $\mathrm{Dj}$. Kurepa in 1963 is very important, where there is result that for every regular cardinal $k \geq \aleph_{0}$ there exists a $k$-metrizable (i.e., an $D_{\alpha}$-space) nonlinearly orderable topological space. A 
proof of this result was exhibit by S. To d o r č e vić in 1981. Also see K u r e p a [27-31].

K a r l M e n g e r initiated the study of probabilistic metric spaces in 1942. A probabilistic metric space in which the "distance" between any two points is a probability distribution function. Every Menger's space is a lower transversal space (see: Tasković [54]). The possibility of defining such notions as limit and continuity in an arbitrary set is an idea which undoubtedly was first put forward by Maurice Fréchet in 1904, and developed by him in his famous doctoral dissertation 1905 (publish in 1906).

In 1934 Djuro Kurepa introduced the notion of a pseudo-metric space: and in 1936 also Dj. Kurepa introduced, for a given ordinal $\alpha$, the notion of $\left(\triangle^{\alpha}\right)$ or $\left(D_{\alpha}\right)$ as the class of pseudo-metric spaces. The case $\alpha=0$ coincides with the class of metric spaces.

A special feature in the former notions (of Fréchet and $\mathrm{Kurepa}$ ) is the "triangular relation" occurring in the elementary geometry and in many other cases.

At the same time, Fréchet consider instead of triangular relation, apparently weaker, regularity condition: There exists a self-map $f$ of $\mathbb{R}_{+}:=(0,+\infty)$ into itself such that $f(x) \rightarrow 0(x \rightarrow 0)$ and that for any triple $(a, b, c)$ of elements of $X$ one has $\rho(a, b)<x$ and $\rho(b, c)<x$ implies $\rho(a, c)<f(x)$.

Fréchet remark that metric spaces $(X, \rho)$ and preceding spaces $(X, \rho, f)$ with the regularity condition have similar properties. In 1910 he asked whether this two classes of spaces should be the same. C h i t t e $\mathrm{n} \mathrm{d} \mathrm{e} \mathrm{n} \mathrm{in} 1917$ confirmed this conjecture. A simple proof was exhibited by $\mathrm{Frin} \mathrm{k}$ in 1937.

We remarked that an important example of upper transversal spaces is also and every Fréchet's space with the regularity condition. For this an upper bisection function $g:\left(\mathbb{R}_{+}^{0}\right) \rightarrow\left(\mathbb{R}_{+}^{0}\right)$ can be defined by $g(s, t)=\max \{x, f(x)\}$.

On the other hand, let $\tau=\omega_{\mu}$ be a regular cardinal number, $X$ a set, and $(G,+, \preccurlyeq)$ a linearly ordered abelian group with cofinality $\operatorname{cof}(G)=\omega_{\mu}$ at the identity element $0 \in G$ (which means that 0 is the infimum of strictly decreasing $\tau$-sequence $\left.\left\{x_{\alpha}: \alpha \in \tau\right\} \subset G \backslash\{0\}\right)$. An $\tau$-metric on $X$ is a function $\rho: X \times X \rightarrow G$ which satisfies all the metric axioms (i.e., $\rho[x, y]=0$ if and only if $x=y, \rho[x, y]=\rho[y, x]$ and $\rho[x, y] \preccurlyeq \rho[x, z]+\rho[z, y])$.

This definition of spaces $X$ was given by R. Sikorski in 1950 using the name $\omega_{\mu}$ metrizable topological space (if its topology can be induced by some $\omega_{\mu}$-metric on $X)$.

Call, for $k=\aleph_{\alpha}(\alpha \geq 0)$, a topological space $X$ a $k$-metrizable space or a $D_{\alpha}$-space if there exist $\rho: X \times X \rightarrow \omega_{\alpha} \cup\left\{\omega_{\alpha}\right\}$ and $\phi: \omega_{\alpha} \rightarrow \omega_{\alpha}$ such that: $\rho(x, y)=\omega_{\alpha}$ if and only if $x=y, \rho(x, y)=\rho(y, x)$, and if $\rho(x, y)>\phi(\xi)$ and $\rho(y, z)>\phi(\xi)$ implies $\rho(x, z)>\xi$. This definition of space $X$ was given by $\mathrm{Dj}$. Kurepa in 1934.

Obviously, $\omega_{\mu}$-metrizable topological spaces are fundamental examples of upper transversal spaces with the upper bisection function $g: G \times G \rightarrow G$ defined by $g(s, t) ;=s+t$.

Also, $D_{\alpha}$-spaces of $\mathrm{Dj}$. Kurepa for $\alpha \geq 0$ are fundamental examples of lower transversal spaces with the lower bisection function $d: P \times P \rightarrow P$ defined by 
$d(s, t):=\inf \{\xi, \phi(\xi)\}$ for some function $\phi: \omega_{\alpha} \rightarrow \omega_{\alpha}$ and $\xi<\omega_{\alpha}$. A fundamental first example of upper transversal space for the upper bisection function $g:\left(\mathbb{R}_{+}^{0}\right)^{2} \rightarrow \mathbb{R}_{+}^{0}$ defined by $g(s, t):=s+t$ is a metric space.

\section{UPPER TRANSVERSES ON PARTIALLY ORDERED INTERVALS}

In connection with the preceding, let $P:=(P, \preccurlyeq)$ be a partially ordered set and for $a, b \in P$ and $a \preccurlyeq b$, the set (interval) $[a, b]$ is defined by

$$
[a, b]:=\{t: t \in P \quad \text { and } \quad a \preccurlyeq t \preccurlyeq b\} .
$$

The function $\rho: X \times X \rightarrow[a, b]$ for $a \preccurlyeq b$ is called an upper ordered interval transverse on $X$ (or upper ordered interval transversal) iff: $\rho[x, y]=\rho[y, x]$ and if there is an upper (ordered interval) bisection function $g:[a, b] \times[a, b] \rightarrow[a, b]$ such that $(\mathrm{A})$.

A transversal upper ordered interval space is a set $X$ together with a given upper ordered interval transverse $\rho: X \times X \rightarrow[a, b]$ for $a \preccurlyeq b$ on $X$, where every decreasing sequence $\left\{u_{n}\right\}_{n \in \mathbb{N}}$ of elements in $[a, b]$ has a unique element $u \in[a, b]$ as limit (in notation $u_{n} \rightarrow u$ or $\lim u_{n}=u$ ).

In further, let $\mathbb{S}$ be a nonempty totally ordered set. A mapping $M: \mathbb{S} \rightarrow$ $[a, b]$ for $a \preccurlyeq b$ is called an upper function if it is decreasing with inf $M=a$ and $\sup M=b$. We will denote by $\mathcal{D}$ the set of all upper functions.

Next two spaces are very interesting examples upper ordered interval spaces.

First, an upper ordered statistical space is a pair $(X, \mathcal{R})$, where $X$ is an abstract set and $\mathcal{R}$ is a mapping of $X \times X$ into the set of all upper functions $\mathcal{D}$. We shall denote the upper function $\mathcal{R}(u, v)$ by $M_{u, v}(x)$ or $M_{u, v}$ whence the symbol $M_{u, v}(x)$ will denote the "value" of $M_{u, v}$ at $x \in \mathbb{S}$. The functions $M_{u, v}$ assumed to satisfy the following conditions: $M_{u, v}=M_{v, u}, M_{u, v}(c)=b$ for some $c \in \mathbb{S}$, and

$$
M_{u, v}(x)=a \text { for } \quad x \succ c \text { if and only if } u=v,
$$

and if $M_{u, r}(x)=a$ and $M_{r, v}(y)=a$ implies $M_{u, v}(\max \{x, y\})=a$ for all $u, v, r \in X$ and for all $x, y \in \mathbb{S}$.

In view of the condition $M_{u, v}(c)=b$, which evidently, implies that $M_{u, v}(c)=b$ for every $x \preccurlyeq c$. Thus condition (Eq) is equivalent to the statement: $u=v$ if and only if $M_{u, v}(x)=A(x)$, where $A(x)=b$ if $x \preccurlyeq c$ and $A(x)=a$ if $x \succ c$.

Also, $M_{u, v}(x)$ may be interpreted as the "measure" that the distance between $u$ and $v$ is less that $x$. Second example of transversal upper ordered interval space, an upper ordered interval space (or Taskovićs ordered interval space from [55]) is a nonempty set $X$ together with the functions $M_{u, v}(x)$ with the following properties: $M_{u, v}=M_{v, u}, M_{u, v}(c)=b$ for some $c \in \mathbb{S},(\mathrm{Eq})$, and if there is a nondecreasing function $f:[a, b] \times[a, b] \rightarrow[a, b]$ with the property $f(t, t) \preccurlyeq t$ for all $t \in[a, b]$ such that

$$
M_{u, v}(\max \{x, y\}) \preccurlyeq f\left(M_{u, r}(x), M_{r, v}(y)\right)
$$

for all $u, v, r \in X$ and for all $x, y \succcurlyeq c$. (Namely, the function $f:[a, b] \times[a, b] \rightarrow[a, b]$ is nondecreasing if $a_{i}, b_{i} \in[a, b]$ and $a_{i} \preccurlyeq b_{i}(i=1,2)$ implies $f\left(a_{1}, a_{2}\right) \preccurlyeq$ $F\left(b_{1}, b_{2}\right)$.) 
We notice, if we had chosen an upper ordered bisection (interval) function $g$ : $[a, b] \times[a, b] \rightarrow[a, b]$ such that $g=f$ (from $(\mathrm{Nt})$ ), then we immediate obtain that every upper ordered interval space, for $\rho[u, v]=M_{u, v}$, is a transversal upper ordered interval space, because in this case from (Nt) for $y=x$ the following inequalities hold:

$$
\begin{gathered}
\rho[u, v]=M_{u, v}(\max \{x, y\}) \preccurlyeq f\left(M_{u, r}(x), M_{r, v}(y)\right):= \\
:=g(\rho[u, r], \rho[r, v]) \preccurlyeq \sup \{\rho[u, r], \rho[r, v], g(\rho[u, r], \rho[r, v])\} .
\end{gathered}
$$

On the other hand, if: $M_{u, v}=M_{v, u}, M_{u, v}(c)=b$ for some $c \in \mathbb{S}$, (Eq), and if there is a function $\psi:[a, b] \times[a, b] \rightarrow[a, b]$ such that

$$
M_{u, v}(x) \preccurlyeq \psi\left(M_{u, r}(x), M_{r, v}(x)\right)
$$

for all $u, v, r \in X$ and for every $x \succcurlyeq c$, then it is an example of transversal upper ordered interval space also.

A very characteristic example, for further work, of the transversal upper ordered interval spaces is the following space in the following form.

A transversal upper ordered interval $T$-space is a pair $(X, \rho)$, where $X$ is a transversal upper ordered interval space and where the upper ordered interval transverse $\rho[u, v]=M_{u, v}(x)$ satisfying: $M_{u, v}=M_{v, u}, M_{u, v}(c)=b$ for some $c \in \mathbb{S}$, and $(\mathrm{Eq})$.

In further, the concept of a neighborhood can ce introduced and defined with the aid of the upper ordered interval transfers. In fact, neighborhoods in transversal upper ordered interval spaces may be defined in several nonequivalent ways. Here we shall consider only one of these.

If $p \in X, \mu \succ c$ for some $c \in \mathbb{S}$ and $r \succ a$, then an $(\mu, r)$-neighborhood of $p$, denoted by $U_{p}(\mu, r)$, is denoted by

$$
U_{p}(\mu, r)=\left\{q \in X: \rho[p, q]=M_{p, q}(\mu) \preccurlyeq r\right\} .
$$

Lemma 1. Let $(X, \rho)$ be a transversal upper ordered interval space, where the upper ordered interval transverse $\rho[p, q]=M_{p, q}(x) . \quad$ If $\varepsilon_{1} \preccurlyeq \varepsilon_{2}$ and $r_{1} \preccurlyeq r_{2}$, then $U_{p}\left(\varepsilon_{1}, r_{1}\right) \subset U_{p}\left(\varepsilon_{2}, r_{2}\right)$.

Proof. Suppose $q \in U_{p}\left(\varepsilon_{1}, r_{1}\right)$ so that $M_{p, q}\left(\varepsilon_{1}\right) \preccurlyeq r_{1}$. Then $M_{p, q}\left(\varepsilon_{2}\right) \preccurlyeq$ $M_{p, q}\left(\varepsilon_{1}\right) \preccurlyeq r_{1} \preccurlyeq r_{2}$, whence, by definition, $q \in U_{p}\left(\varepsilon_{2}, r_{2}\right)$. The proof is complete.

From the preceding facts, in this topology a sequence $\left\{p_{n}\right\}_{n \in \mathbb{N}}$ in $X$ converges to a point $p \in X$ (in notation $p_{n} \rightarrow p$ ) if for some $c \in \mathbb{S}$ and for every $\mu \succ c$ and every $\sigma \succ a$, there exists an integer $\mathcal{M}(\mu, \sigma)$ such that $p_{n} \in U_{p}(\mu, \sigma)$, i.e., $\rho\left[p, p_{n}\right]=M_{p, p_{n}}(\mu) \preccurlyeq \sigma$ whenever $n \geq \mathcal{M}(\mu, \sigma)$.

Lemma 2. Let $(X, p)$ be a transversal upper ordered interval T-space, where the upper transverse $\rho[u, v]=M_{u, v}(x)$. If $p_{n} \rightarrow p$, then $M_{p, p_{n}} \rightarrow M_{p, p}=A$, i.e., for every $x \in \mathbb{S}, M_{p, p_{n}}(x) \rightarrow M_{p, p}(x)=A(x)$, and conversely.

Proof. If $x \succ c$, then for every $\varepsilon \succ a$, there exists an integer $\mathcal{M}(x, \varepsilon)$ such that $M_{p, p_{n}}(x) \preccurlyeq \varepsilon$ whenever $n \geq \mathcal{M}(x, \varepsilon)$. But this means that 
$\lim _{n \rightarrow \infty} M_{p, p_{n}}(x)=a=M_{p, p}(x)$. If $x=c$, then for every $n, M_{p, p_{n}}(c)=b$ and hence $\lim _{n \rightarrow \infty} M_{p, p_{n}}(c)=b=M_{p, p}(c)$. The converse is immediate. The proof is complete.

On the other hand, if $T: X \rightarrow X$, then a function $u \mapsto M_{u, T u}(x)$ is ordered lower semicontinuous at $\xi \in X$ if $\left\{u_{n}\right\}_{n \in \mathbb{N}}$ is a sequence in $X$ and $u_{n} \rightarrow \xi(n \rightarrow \infty)$ implies that $M_{\xi, T \xi} \preccurlyeq \lim M_{u_{n}, T u_{n}}(x)$ for every $x \in \mathbb{S}$.

In connection with the preceding, the sequence $\left\{p_{n}\right\}_{n \in \mathbb{N}}$ will be called fundamental in $X$ if for some $c \in \mathbb{S}$ and for each $\mu \succ c, \sigma \succ a$ there is an integer $\mathcal{M}(\mu, \sigma)$ such that $\rho\left[p_{n}, p_{m}\right]=M_{p_{n}, p_{m}}(\mu) \preccurlyeq \sigma$ whenever $n, m \geq$ $\mathcal{M}(\mu, \sigma)$, or in an equivalent entry as

$$
\lim _{n \rightarrow \infty}\left(\operatorname{diam}\left\{p_{k}: k \geqslant n\right\}\right)=a .
$$

In analogy with the completion concept of metric space, a transversal upper ordered interval space $X$ will be called complete if each fundamental sequence in $X$ converges to an element in $X$.

In further we introduce a notion of an ordered interval upper contraction on a transversal upper ordered interval space and prove a fixed point statement.

A mapping $T$ of a transversal ordered upper interval space $(X, \rho)$ into itself for $\rho[u, v]=M_{u, v}(x)$ will be called an ordered interval upper contraction in there exists an increasing function $\varphi: \mathbb{S} \rightarrow \mathbb{S}$ such that $t \preccurlyeq \varphi(t)$ for every $t \in \mathbb{S}$, for some $c \in \mathbb{S}$ that is

$$
\lim _{n \rightarrow \infty} M_{u, v}\left(\varphi^{n}(t)\right)=a \quad \text { for every } \quad t \succ c,
$$

and such that

$$
\begin{gathered}
M_{T u, T v}(x) \preccurlyeq \\
\preccurlyeq \sup \left\{M_{u, v}(\varphi(x)), M_{u, T u}(\varphi(x)), M_{v, T v}(\varphi(x)), M_{u, T v}(\varphi(x)), M_{v, T u}(\varphi(x))\right\}
\end{gathered}
$$

for all $u, v \in X$ and for every $x \succ c$.

Theorem 1. Let $(X, \rho)$ be a complete transversal ordered upper interval $T$-space, where the upper transverse $\rho[u, v]=M_{u, v}(x)$ is ordered lower semicontinuous and the upper bisection function $g:[a, b] \times[a, b] \rightarrow[a, b]$ is nondecreasing such that $g(t, t) \preccurlyeq t$ for all $t \in[a, b]$. If $T$ is any ordered interval upper contraction mapping of $X$ into itself, then there is a unique point $p \in X$ such that $T p=p$. Moreover, $T^{n} q \rightarrow p$ for each $q \in X$.

Proof. For this proof the following inequalities are essential. Namely, from the conditions for the function $g:[a, b] \times[a, b] \rightarrow[a, b]$ we obtain the following inequalities

$$
g(s, t) \preccurlyeq g(\sup \{s, t\}, \sup \{s, t\}) \preccurlyeq \sup \{s, t\}
$$

for all $s, t \in[a, b]$. On the other hand, since $X$ is a transversal ordered upper interval space, for some $c \in \mathbb{S}$ and for every $x \succcurlyeq c$ we have from (1) 
the following inequalities

$$
\begin{gathered}
M_{u, v}(x) \preccurlyeq \sup \left\{M_{u, r}(x), M_{r, v}(x), g\left(M_{u, r}(x), M_{r, v}(x)\right)\right\} \\
\preccurlyeq \ldots \preccurlyeq \sup \left\{M_{u, r}(x), M_{r, v}(x)\right\} .
\end{gathered}
$$

To prove the existence of the fixed point, consider an arbitrary $u \in X$, and define $u_{n}=T^{n}(u)$, for $n \in \mathbb{N} \cup\{0\}$. We show that sequence $\left\{u_{n}\right\}_{n \in \mathbb{N} \cup\{0\}}$ is fundamental in $X$. Then for $t \succ c$ and $m>n(m, n \in \mathbb{N})$ from (2) is

$$
M_{u_{n}, u_{m}}(t) \preccurlyeq \sup \left\{M_{u_{n}, u_{n+1}}(t), \ldots, M_{u_{m-1}, u_{m}}(t)\right\} .
$$

On the other hand, since $T$ is an ordered interval upper contraction mapping, from (2) we obtain for every $t \succ c$ the following inequalities

$$
\begin{gathered}
M_{u_{n}, u_{n+1}}(t)=M_{T u_{n-1}, T u_{n}}(t) \preccurlyeq \\
\preccurlyeq \sup \left\{M_{u_{n-1}, u_{n}}(\varphi(t)), M_{u_{n-1}, T u_{n-1}}(\varphi(t)),\right. \\
\left.M_{u_{n-1}, T u_{n}}(\varphi(t)), M_{u_{n}, T u_{n-1}}(\varphi(t)), M_{u_{n-1}, T u_{n}}(\varphi(t))\right\}= \\
=\sup \left\{M_{u_{n-1}, u_{n}}(\varphi(t)), M_{u_{n-1}, u_{n}}(\varphi(t)), M_{u_{n}, u_{n+1}}(\varphi(t)),\right. \\
\left.M_{u_{n}, u_{n}}(\varphi(t)), M_{u_{n-1}, u_{n+1}}(\varphi(t))\right\} \preccurlyeq \\
\preccurlyeq \sup \left\{M_{u_{n-1}, u_{n}}(\varphi(t)), M_{u_{n}, u_{n+1}}(\varphi(t)), M_{u_{n-1}, u_{n+1}}(\varphi(t))\right\} \preccurlyeq \\
\preccurlyeq \sup \left\{M_{u_{n-1}, u_{n}}(\varphi(t)), M_{u_{n}, u_{n+1}}(\varphi(t))\right\}
\end{gathered}
$$

and thus

$$
M_{u_{n}, u_{n+1}}(\varphi(t)) \preccurlyeq \sup \left\{M_{u_{n-1}, u_{n}}\left(\varphi^{2}(t)\right), M_{u_{n}, u_{n+1}}\left(\varphi^{2}(t)\right)\right\} .
$$

From (4) and (5) it follows by induction that for every integer $k \in \mathbb{N}$ the following inequality holds

$$
M_{u_{n}, u_{n+1}}(t) \preccurlyeq \sup \left\{M_{u_{n-1}, u_{n}}(\varphi(t)), M_{u_{n}, u_{n+1}}\left(\varphi^{k}(t)\right)\right\},
$$

that is, when $k \rightarrow \infty$, we obtain $M_{u_{n}, u_{n+1}}(t) \preccurlyeq M_{u_{n-1}, u_{n}}(\varphi(t))$ for every $n \in \mathbb{N}$, i.e.,

$$
M_{u_{n}, u_{n+1}}(t) \preccurlyeq M_{u_{0}, u_{1}}\left(\varphi^{n}(t)\right)
$$

for every $n \in \mathbb{N}$. Hence, from the former inequality (3), we obtain

$$
M_{u_{n}, u_{m}}(t) \preccurlyeq \sup \left\{M_{u_{0}, u_{1}}\left(\varphi^{n}(t)\right), \ldots, M_{u_{0}, u_{1}}\left(\varphi^{m-1}(t)\right)\right\}=M_{u_{0}, u_{1}}\left(\varphi^{n}(t)\right),
$$

that is, $M_{u_{n}, u_{m}}(t) \preccurlyeq M_{u_{0}, u_{1}}\left(\varphi^{n}(t)\right)$. Hence, since $\left\{M_{u_{0}, u_{1}}\left(\varphi^{n}(t)\right)\right\}_{n \in \mathbb{N}}$ is a decreasing sequence from definition of the limes on $[a, b]$ and (As), $\left\{u_{n}\right\}_{n \in \mathbb{N} \cup\{0\}}$ is a fundamental sequence in $X$. Since $X$ is a complete space, there is an $p \in X$ such that $u_{n} \rightarrow p$, that is $T^{n}(u) \rightarrow p$. 
Since $\rho[u, v]=M_{u, v}(t)$ is an ordered lower semicontinuous function, from (As) and (6), we have

$M_{p, T p}(t) \preccurlyeq \lim M_{u_{n}, T\left(u_{n}\right)}(t)=\lim M_{u_{n}, u_{n+1}}(t) \preccurlyeq \ldots \preccurlyeq \lim M_{u_{0}, u_{1}}\left(\varphi^{n}(t)\right)=a$, i.e., this means that is $T p=p$.

We further prove the uniqueness. Suppose $p \neq q$ and $T p=p, T q=q$. Then, there exists an $x \succ c$ and an $a \preccurlyeq r \preccurlyeq b$, such that $M_{p, q}(x)=r$. However, since $T$ is an ordered interval upper contraction mapping, we have

$$
\begin{gathered}
M_{p, q}(x)=M_{T p, T q}(x) \preccurlyeq \\
\preccurlyeq \sup \left\{M_{p, q}(\varphi(x)), M_{p, p}(\varphi(x)), M_{q, q}(\varphi(x)), M_{p, q}(\varphi(x)), M_{q, p}(\varphi(x))\right\}
\end{gathered}
$$

and thus $M_{p, q}(x) \preccurlyeq M_{p, q}(\varphi(x))$, i.e., by induction,

$$
r=M_{p, q}(x) \preccurlyeq M_{p, q}(\varphi(x)) \preccurlyeq \ldots \preccurlyeq M_{p, q}\left(\varphi^{n}(x)\right) ;
$$

and hence, since $M_{p, q}\left(\varphi^{n}(x)\right) \rightarrow a$ as $n \rightarrow \infty$, it follows that $r=a$, i.e., $p=q$. This contradicts the choice of $a \preccurlyeq r \preccurlyeq b$, and therefore, the fixed point is unique. The proof is complete.

In connection with the preceding statement, from our the Principle of Symmetry (see: Math. Japonica, 35 (1990), p. 661), we obtain as an immediate consequence of Theorem 1 the following result.

Theorem 2. Let $(X, \rho)$ be a complete transversal ordered upper interval $T$-space, where the upper transverse $\rho[u, v]=M_{u, v}(x)$ is ordered lower semicontinuous and the upper bisection function $g:[a, b] \times[a, b] \rightarrow[a, b]$ is nondecreasing such that $g(t, t) \preccurlyeq t$ for all $t \in[a, b]$. If there exists an increasing function $\varphi: \mathbb{S} \rightarrow \mathbb{S}$ such that $t \preccurlyeq \varphi(t)$ for every $t \in \mathbb{S}$ with the property $(A s)$ for some $c \in \mathbb{S}$ and if for each $u \in X$ there is a positive integer $n=n(u)$ such that

$$
M_{T^{n}(u), T^{n}(v)}(x) \preccurlyeq
$$

$\preccurlyeq \sup \left\{M_{u, v}(\varphi(x)), M_{u, T^{n} u}(\varphi(x)), M_{v, T^{n} v}(\varphi(x)), M_{u, T^{n} v}(\varphi(x)), M_{v, T^{n} u}(\varphi(x))\right\}$

for every $v \in X$ for every $x \succ c$, then $T$ has exactly one fixed point $p \in X$ and $T^{n} q \rightarrow p$ for every $q \in X$.

On the other hand, from the preceding facts, since every upper ordered interval space is a transversal upper ordered interval $T$-space, hence Theorems 1 and 2 holds and for upper ordered interval spaces; similar, and for upper ordered statistical spaces.

Also, as an immediate consequence of the preceding Theorem 1 we obtain directly the following interesting cases of upper ordered interval contractive mappings:

(M) There exists an increasing function $\varphi: \mathbb{S} \rightarrow \mathbb{S}$ with the property (As) for some $c \in \mathbb{S}$ such that for each $u, v \in X$ and for every $x \succ c$ the following inequality holds

$$
M_{T u, T v}(x) \preccurlyeq M_{u, v}(\varphi(x)) .
$$


If the interval $[a, b]$ for $a \preccurlyeq b$ is a totally ordered set, then as a directly consequence of the Theorem 1 we obtain the following case of upper ordered interval contractive mappings:

$(\mathrm{N})$ There is an increasing function $\varphi: \mathbb{S} \rightarrow \mathbb{S}$ such that $t \preccurlyeq \varphi(t)$ for every $t \in \mathbb{S}$ with the property (As) and for all $u, v \in X$ and for every $x \succ c$ that is

$$
\begin{gathered}
M_{T u, T v}(x) \preccurlyeq \\
\preccurlyeq \max \left\{M_{u, v}(\varphi(x)), M_{u, T u}(\varphi(x)), M_{v, T v}(\varphi(x)), M_{u, T v}(\varphi(x)), M_{v, T u}(\varphi(x))\right\} .
\end{gathered}
$$

If $[a, b] \subset \mathbb{R}_{+}^{0}:=[0,+\infty)$ for $a<b$ and $\mathbb{S}=\mathbb{R}$, then we obtain the following two cases of upper ordered interval contractive mappings:

(S) There exists a constant $0<k<1$ such that for each $p, q \in X$, for some $c \in \mathbb{R}$ and for every $x>c$ the following inequality holds

$$
M_{T p, T q}(x) \leq M_{p, q}\left(\frac{x}{k}\right) .
$$

(R) There exists a constant $0<k<1$ such that for each $u, v \in X$, for some $c \in \mathbb{R}$ and for every $x>c$ the following inequality holds

$$
M_{T u, T v}(k x) \leq \max \left\{M_{u, v}(x), M_{u, T u}(x), M_{v, T v}(x), M_{u, T v}(x), M_{v, T u}(x)\right\} .
$$

Annotations. In connection with the preceding facts, we notice that third characteristic example of a transversal upper ordered interval space is so-called upper edges ordered space of the form $(X, \rho)$, where $X$ is a nonempty set and $\rho: X \times X \rightarrow[a, b)$ for $a \preccurlyeq b$ is given upper edges ordered transverse with the properties: $\rho[x, y]=a$ if and only if $x=y$, $\rho[x, y]=\rho[y, x]$, and if there is a function $\psi:[a, b)^{2} \rightarrow[a, b)$ such that

$$
\rho[x, y] \preccurlyeq \sup \{\rho[x, z], \rho[z, y], \psi(\rho[x, z], \rho[z, y])\}
$$

for all $x, y, z \in X$. In this sense, an example, we can suppose that every decreasing sequence of elements in $[a, b)$ has a unique element as limit in $[a, b)$.

In this sense, in upper edges ordered space $(X, \rho)$, the sequence $\left\{x_{n}\right\}_{n \in \mathbb{N}}$ upper converges (or upper edges ordered converges) to a point $x \in X$ (in notation $\left.x_{n} \rightarrow x(n \rightarrow \infty)\right)$ if for some decreasing sequence $\left\{a_{n}\right\}_{n \in \mathbb{N}}$ in $[a, b)$ which converges to $a$ the following inequality holds in the form

$$
\rho\left[x, x_{n}\right] \preccurlyeq a_{n} \quad \text { for every } n \in \mathbb{N},
$$

or for $n$ large enough.

On the other hand, in connection with this, the sequence $\left\{x_{n}\right\}_{n \in \mathbb{N}}$ in $X$ will be called upper fundamental (or upper edges ordered fundamental) if the following inequality holds in the form

$$
\rho\left[x_{n}, x_{m}\right] \preccurlyeq a_{n} \text { for all } n, m \in \mathbb{N} \quad(n<m),
$$

or for $n$ and $m$ large enough, where the decreasing sequence $\left\{a_{n}\right\}_{n \in \mathbb{N}}$ in $[a, b)$ converges to $a$. 
An upper edges ordered space $(X, \rho)$ is called upper complete (or upper edges ordered complete) if any upper fundamental sequence $\left\{x_{n}\right\}_{n \in \mathbb{N}}$ in $X$ is upper convergent (to a point of $X$, of course). For further results on upper edges ordered spaces see: Tasković [57].

Examples of the transversal upper edges ordered spaces. In the next section of this part paper we give some illustrations of the upper edges ordered spaces and the set $[a, b)$.

Example 1. (K u r e p a [27]). As a first example of upper edges ordered spaces we obtain so-called pseudodistantial space by $Đ$. Kurepa with the nonnumerical transverse (of the form) for the bisection function $g(s, t)=s+t$. In this case the nonnumerical transverse is given in the partially ordered set $[a, b)$, where the element $a$ in the partially ordered set $G$ of the following form: $[a, b)=(G,+, \preccurlyeq)$ is a linearly ordered abelian groups with cofinality $\operatorname{cof}(G)=w_{\mu}$ at he identity element $a=\theta \in G$ (which means that $a$ is the infimum of a strictly decreasing $\tau$-sequnce. An $\tau$-metric on $X$ is a function $\rho: X \times X \rightarrow[a, b)=G$ which satisfies all the metric axioms (i.e., $\rho[x, y]=\theta=a$ if and only if $x=y, \rho[x, y]=\rho[y, x]$, and $\rho[x, y] \preccurlyeq \rho[x, z]+\rho[z, y]$ ). For this see: Collatz [11], C a m marotoK o čin ac [17] and Sikorski [50].

Example 2. (Kantorovitch's lineal, $\mathrm{K}$ a n t o r o v it c h [26]). Let $K$ be a real linear space and $K$ be a $K$-lineal, by L.V. Kantorovitch. In this sense, $K$ is a K-lineal if there exists an element $\theta \in K$ as a neutral element such that $\theta \preccurlyeq x$ for all $x \in K$, and if the partially ordering $\preccurlyeq$ is defined with $x \preccurlyeq y$ if and only if $x-y \succcurlyeq \theta$ for all $x, y \in K$ such that the following hold:

(i) $x \succ \theta, y \succ \theta$ implies $x \neq \theta, x+y \succ \theta$, and $x y \succ \theta$;

(ii) there exists $\sup \{x, y\}$ for two arbitrary elements $x, y \in K$.

In this case, $[a, b)=K$ with nonnumerical upper transversal $\rho: X \times X \rightarrow K$ as an element in $K$.

Example 3. (M-sets, Tasković [51]). Let $\mathcal{O}$ be a partially ordered set by the relation $\preccurlyeq$ such that there exists $\theta \in \mathcal{O}$ with the property: a) $\theta \preccurlyeq u$ for every $u \in \mathcal{O}$. Also, 2): for every nonincreasing sequence $\left\{u_{n}\right\}_{n \in \mathbb{N}}$ there exists the uniques element $u \in \mathcal{O}$ called the limit of $\left\{u_{n}\right\}_{n \in \mathbb{N}}$ all signed by $u=\lim _{n \rightarrow \infty} u_{n}$ (alternative designation $u_{n} \downarrow u$ ) such that: $u_{n}=u$ (for $n \in \mathbb{N}$ ) implies $u_{n} \downarrow u$; if $u_{n} \downarrow u, v_{n} \downarrow v, u_{n} \preccurlyeq v_{n}$ then $u \preccurlyeq v$; and the limit of $\left\{u_{n}\right\}_{n \in \mathbb{N}}$ is invariant with respect to the initial conditions. The partially ordered set $\mathcal{O}$ with the preceding properties we call the M-set.

We notice that the property 2 ) is specially realized if in $\mathcal{O}$ is introduced the usual ordered topological structure and eachy subset of $\mathcal{O}$ from the upper side bounded has its supremum, the term of limit having its standard meaning.

If the set $[a, b)=\mathcal{O}$ where $a=\theta$ we obtain an upper nonnumerical transverse $\rho: X \times X \rightarrow \mathcal{O}$ as an element in $[a, b)$. It is evident that $\rho$ satisfy all conditions of the upper transversal ordered edges transverse!!

Example 4. (The cone metrical spaces, $\mathrm{K}$ u $\mathrm{r}$ e p a [30]). We begin by introducing a structure ot the ordered Banach space and define a cone metric space. Let $E$ be a real Banach space and $P$ a subset of $E$. In this case $P$ is called a cone if: $P$ is a closed, nonempty set and $P \neq\{0\}, a x+b y \in P$ for all $a, b \in \mathbb{R}(a, b \geq 0)$ and $x, y \in P$, and $x \in P$ such that $-x \in P$ implies $x=0$. In this case $a=0 \in[a, b)$. 
Given a cone $P \subset E$ we define a partially ordering $\preccurlyeq$ with respect to $P$ by $x \preccurlyeq y$ if and only if $y-x \in P$. We shall write $x \preccurlyeq y$ iff $x \preccurlyeq y$ and $x \neq y$. Also, we shall write $x \ll y$ iff $y-x \in \operatorname{Int} P$, where Int $P$ denotes the interior of $P$.

In this sense, suppose that the mapping $\rho: X \times X \rightarrow E$ has all the metric axioms as in the papers: K u r e p a [30], Collatz [11] and Sikorski [50]. Thus $\rho$ satisfis all the axioms of the upper transversal ordered edges spaces (as and all axioms of Kurepa's pseudodistantial spaces, see: K u r e p a [30]).

Equations on edges ordered spaces. In connection with the edges ordered spaces we have the following applications. Let $P:=(P, \preccurlyeq)$ be a partially ordered set, and let $\left\{u_{n}^{i}\right\}_{n \in \mathbb{N}}$ for $i=1, \ldots, k$ be sequences in $[a, b) \subset P(a \preccurlyeq b)$ such that monotone decreasing converges to $u^{i} \in[a, b)$ for $i=1, \ldots, k$.

In this context, if $f:[a, b)^{k} \rightarrow[a, b)$, for the fixed $k \in \mathbb{N}$, satisfying that $f\left(u_{n}^{1}, \ldots, u_{n}^{k}\right)$ monotone decreasing converges to $f\left(u^{1}, \ldots, u^{k}\right)$ as $n \rightarrow \infty$, then we say that $f$ is continuous with respect to sequences. The following result provided by Tasković [51].

Theorem 3. Let $(X, \rho)$ be an upper edges ordered space with the continuous upper transverse $\rho: X \times X \rightarrow[a, b)$ for $a \preccurlyeq b$ and let the upper bisection function $\psi:[a, b)^{2} \rightarrow[a, b)$ is nondecreasing such that $\psi(t, t) \preccurlyeq t$ for all $t \in[a, b)$. Let $X$ be an upper complete space, and let $K:=K(z, r) \subset X$ be a sphere. If $T: X^{k} \rightarrow X$ (for a fixed $k \in \mathbb{N}$ ), and if there exists a continuous nondecreasing function $f$ : $[a, b)^{k} \rightarrow[a, b)$ satisfying

$$
\rho\left[T\left(u_{1}, \ldots, u_{k}\right), T\left(v_{1}, \ldots, v_{k}\right)\right] \preccurlyeq f\left(\rho\left[u_{1}, v_{1}\right], \ldots, \rho\left[u_{k}, v_{k}\right]\right)
$$

for all $u_{i}, v_{i} \in K(i=1, \ldots, k)$ such that the equation $x=f(x, \ldots, x)$ for $x \in[a, b)$ has the unique solution $a$. If there exists $q \in[a, b)$ such that

$$
\rho[z, T(z, \ldots, z)] \preccurlyeq q \quad \text { and } \quad \sup \{q, f(r, \ldots, r)\} \preccurlyeq r,
$$

where $f(y, \ldots, y) \preccurlyeq y$ for $r \preccurlyeq y$, then the following facts hold in suitable form as:

1) $T(\xi, \ldots, \xi)=\xi$ for some $\xi \in K(z, r)$.

2) The point $\xi \in K(z, r)$ is the limit of the sequence $\left\{x_{n}\right\}_{n \in \mathbb{N}}$ in the space $X$ defined by the following equalities of the form as

$$
x_{n+k}=T\left(x_{n}, \ldots, x_{n+k-1}\right) \quad \text { for all } n \in \mathbb{N},
$$

where the points $x_{1}, \ldots, x_{k} \in K(z, r)$ are arbitrarily chosen. In this context, the unique solution of the following equation of the form as,

$$
x=T(x, \ldots, x) \quad \text { for } \quad x \in X,
$$

is the point $\xi=\lim _{n \rightarrow \infty} x_{n}$ and holds the following inequality in the form as $\rho\left[x_{n+k}, \xi\right] \preccurlyeq A_{n+k}(y)$, where $A_{i}(y):=f_{i}(y, \ldots, y)=y$ for $i \leqslant k$ and for $i>k$ that is

$$
A_{i}(y):=f_{i}(y, \ldots, y)=f\left(f_{i-k}(y, \ldots, y), \ldots, f_{i-1}(y, \ldots, y)\right) .
$$

Some annotations. In the Figure 1(i) we give the geometric interpretation of the preceding localization statement which is realized by the condition (A') on a 
sphere $K$. On the other hand, in 1977 we have a localization condition of the form as

$$
\rho\left[T\left(u_{1}, \ldots, u_{k}\right), T\left(u_{2}, \ldots, u_{k+1}\right)\right] \preccurlyeq f\left(\rho\left[u_{1}, u_{2}\right], \ldots, \rho\left[u_{k}, u_{k+1}\right]\right)
$$

for all points $u_{1}, \ldots, u_{k+1} \in X$, where all other facts of Theorem 3 hold. For (B') we give a geometric interpretation on Figure 1(ii) also. In connection with the preceding facts see: Waž e w s ki [61], K u r p elj [15], and Taskovi ć [51].

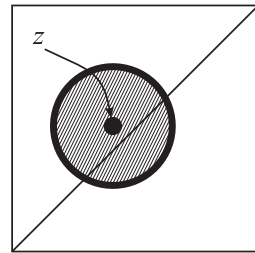

(i)

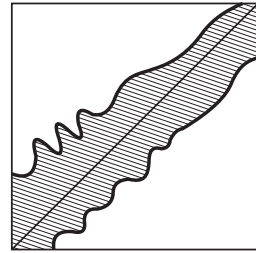

(ii)

FiguRE 1.

Also, we notice that under the preceding conditions of Theorem 3 substituting the condition $\left(\mathrm{A}^{\prime}\right)$ with the following condition of the form as

$$
\rho\left[T_{n}\left(u_{1}, \ldots, u_{k}\right), T_{n+1}\left(v_{1}, \ldots, v_{k}\right)\right] \preccurlyeq f\left(\rho\left[u_{1}, v_{1}\right], \ldots, \rho\left[u_{k}, v_{k}\right]\right),
$$

for all $u_{i}, v_{i} \in K(z, r)$ with $i=1, \ldots, k$ and where $T_{n}: X^{k} \rightarrow X(k \in \mathbb{N}$ is fixed) for $n \in \mathbb{N}$ is a sequence of functions, and $\rho[z, T(z, \ldots, z)] \preccurlyeq q$ with $\rho\left[z, T_{n}(z, \ldots, z)\right] \preccurlyeq$ $q$; affirmation of Theorem 3 is valuable, but relates to the sequence of the form as

$$
x_{n+k}=T_{n}\left(x_{n}, \ldots, x_{n+k-1}\right) \text { for } n \in \mathbb{N} .
$$

Proof of Theorem 3. Let $K:=K(z, r) \subset X$ be a sphere and let $u_{1}, \ldots, u_{k} \in$ $K(z, r)$ be arbitrary points. Since $X$ is an upper edges transversal ordered space and $\psi$ is increasing, then we have the following inequalities in the form as

$$
\begin{gathered}
\rho\left[T\left(u_{1}, \ldots, u_{k}\right), z\right] \preccurlyeq \\
\preccurlyeq \sup \left\{\rho\left[T\left(u_{1}, \ldots, u_{k}\right), T(z, \ldots, z)\right], \rho[T(z, \ldots, z), z],\right. \\
\left.\psi\left(\rho\left[T\left(u_{1}, \ldots, u_{k}\right), T(z, \ldots, z)\right], \rho[T(z, \ldots, z), z]\right)\right\} \preccurlyeq \\
\ldots \ldots \ldots \ldots \ldots \ldots \ldots \\
\preccurlyeq \sup \left\{f\left(\rho\left[u_{1}, z\right], \ldots, \rho\left[u_{k}, z\right]\right), q, \psi\left(f\left(\rho\left[u_{1}, z\right], \ldots, \rho\left[u_{k}, z\right]\right), q\right)\right\} \preccurlyeq \\
\preccurlyeq \sup \{f(r, \ldots, r), q, \psi(f(r, \ldots, r), q)\} \preccurlyeq \sup \{f(r, \ldots, r), q\} \preccurlyeq r,
\end{gathered}
$$

we conclude that the mapping $T$ maps $(K(z, r))^{k}$ into $K(z, r)$. Let $x_{n} \in K(z, r)$ for $n \in \mathbb{N}$. Then we obtain the following inequalities in the form as

$$
\begin{aligned}
& \rho\left[x_{i}, x_{m+i}\right] \preccurlyeq \sup \left\{\rho\left[x_{i}, z\right], \rho\left[z, x_{m+i}\right], \psi\left(\rho\left[x_{i}, z\right], \rho\left[z, x_{m+i}\right]\right)\right\} \preccurlyeq \\
& \preccurlyeq \sup \{r, r, \psi(r, r)\} \preccurlyeq \sup \{r, r, r\}=r \preccurlyeq y
\end{aligned}
$$


for $m \in \mathbb{N}$ and for $i=1, \ldots, k$. On the other hand, we supposed that the following fact holds in the following form as

$$
\rho\left[x_{n+i}, x_{n+m+i}\right] \preccurlyeq f_{n+i}(y, \ldots, y):=A_{n+i}(y)
$$

for $i=1, \ldots, k$ and where $f_{i}(y, \ldots, y)=y:=A_{i}(y)$ for $i \leqslant k$, and where the following convention holds in the suitable form as

$$
f_{i}(y, \ldots, y)=f\left(f_{i-k}(y, \ldots, y), \ldots, f_{i-1}(y, \ldots, y)\right):=A_{i}(y)
$$

for $i>k$; i.e., then in the context of the preceding facts we obtain the following inequalities in the form as

$$
\begin{gathered}
\rho\left[x_{n+k}, x_{n+k+m+1}\right]=\rho\left[T\left(x_{n+1}, \ldots, x_{n+k}\right), T\left(x_{n+m+1}, \ldots, x_{n+k+m}\right)\right] \preccurlyeq \\
\preccurlyeq f\left(\rho\left[x_{n+1}, x_{n+m+1}\right], \ldots, \rho\left[x_{n+k}, x_{n+m+k}\right]\right) \preccurlyeq \\
\preccurlyeq f\left(f_{n+1}(y, \ldots, y), \ldots, f_{n+k}(y, \ldots, y)\right):=f_{n+k+1}(y, \ldots, y):=A_{n+k+1}(y),
\end{gathered}
$$

and thus (7) holds for every $n \in \mathbb{N}$. Also, we have $A_{1}(y)=\cdots=A_{k}(y)=y$. If we suppose that $A_{n+i}(y) \preccurlyeq A_{n+i-1}($ for $i=1, \ldots, k)$, then

$$
A_{n+k+1}(y)=f\left(A_{n+1}(y), \ldots, A_{n+k}(y)\right) \preccurlyeq f\left(A_{n}(y), \ldots, A_{n+k-1}(y)\right)=A_{n+k}(y) ;
$$

therefore, the sequence $\left\{A_{n}(y)\right\}_{n \in \mathbb{N}}$ is nonincreasing such that $a \preccurlyeq A_{n}(y)$, i.e., this sequence is convergent. Let $A_{n}(y) \rightarrow \alpha(n \rightarrow \infty)$. Then $\alpha$ satisfies the equation $x=f(x, \ldots, x)$, i.e., $\alpha=a$. Hence, by upper completeness of $X$, there is an $\xi \in X$ such that $\xi=\lim _{n \rightarrow \infty} x_{n} \in K(z, r)$. Making $m \rightarrow \infty$ in (7) we get $\rho\left[x_{n+k}, \xi\right] \preccurlyeq A_{n+k}(y)$. On the other hand, we have

$$
\begin{aligned}
& \rho[\xi, T(\xi, \ldots, \xi)] \preccurlyeq \\
& \preccurlyeq \sup \left\{\rho\left[\xi, x_{n+k}\right], \rho\left[x_{n+k}, T(\xi, \ldots, \xi)\right], \psi\left(\rho\left[\xi, x_{n+k}\right], \rho\left[x_{n+k}, T(\xi, \ldots, \xi)\right]\right)\right\} \preccurlyeq \\
& \preccurlyeq \sup \left\{\rho\left[\xi, x_{n+k}\right], f\left(\rho\left[x_{n}, \xi\right], \ldots, \rho\left[x_{n+k-1}, \xi\right]\right),\right. \\
& \left.\psi\left(\rho\left[\xi, x_{n+k}\right], f\left(\rho\left[x_{n}, \xi\right], \ldots, \rho\left[x_{n+k-1}, \xi\right]\right)\right)\right\} \preccurlyeq \\
& \preccurlyeq \sup \left\{A_{n+k}(y), f\left(A_{n}(y), \ldots, A_{n+k-1}(y)\right),\right. \\
& \left.\psi\left(A_{n+k}(y), f\left(A_{n}(y), \ldots, A_{n+k-1}(y)\right)\right)\right\} \preccurlyeq \\
& \preccurlyeq \sup \left\{A_{n+k}(y), f\left(A_{n}(y), \ldots, A_{n+k-1}(y)\right)\right\} \preccurlyeq A_{n+k}(y) \text {; }
\end{aligned}
$$

hence, for $n \rightarrow \infty$ we get that $\xi$ satisfies the equation $x=T(x, \ldots, x)$. If $\eta \in K(z, r)$ is an element satisfying $\eta=T(\eta, \ldots, \eta)$, then

$\rho\left[\eta, x_{i}\right] \preccurlyeq \sup \left\{\rho[\eta, z], \rho\left[z, x_{i}\right], \psi\left(\rho[\eta, z], \rho\left[z, x_{i}\right]\right)\right\} \preccurlyeq \sup \{r, r, \psi(r, r)\} \preccurlyeq r \preccurlyeq y=A_{i}(y)$ 
for $i=1, \ldots, k$. If the following inequality holds in the form $\rho\left[\eta, x_{n+i}\right] \preccurlyeq A_{n+i}(y)$ for $i=1, \ldots, k$, then the following inequalities hold in the form as

$$
\begin{gathered}
\rho\left[\eta, x_{n+k+1}\right]=\rho\left[T(\eta, \ldots, \eta), T\left(x_{n+1}, \ldots, x_{n+k}\right)\right] \\
\preccurlyeq f\left(\rho\left[\eta, x_{n+1}\right], \ldots, \rho\left[\eta, x_{n+k}\right]\right) \preccurlyeq f\left(A_{n+1}(y), \ldots, A_{n+k}(y)\right)=A_{n+k+1}(y) ;
\end{gathered}
$$

which means that $a \preccurlyeq \rho\left[\eta, x_{n+i}\right] \preccurlyeq A_{n+i}(y)$ for every $n \in \mathbb{N}$. Hence, as $n \rightarrow \infty$, we obtain the following inequalities $a \preccurlyeq \rho[\eta, \xi] \preccurlyeq a$, i.e., $\eta=\xi$, because $X$ is an upper edges transversal ordered space. The proof is complete.

Open problem 16. Let $(X, \rho)$ be a lower edges transversal ordered space with the lower bisection function $d:(a, b]^{2} \rightarrow(a, b]$ for $a \preccurlyeq b(a, b \in P:=(P, \preccurlyeq))$. If $T: X^{k} \rightarrow X(k \in \mathbb{N}$ is fixed $)$, to invent suitable sufficient conditions for solutions of the following equation in the form as

$$
x=T(x, \ldots, x) \quad \text { for } \quad x \in X
$$

on the lower edges transversal ordered space $X$, does possible some condition of the symmetric form from (A')? To invent a suitable statement on the lower edges transversal ordered space?!

\section{LOWER TRANSVERSES ON PARTIALLY ORDERED INTERVALS}

In connection with with the preceding, the function $\rho: X \times X \rightarrow[a, b]$ for $a \preccurlyeq b$ is called a lower ordered interval transverse on $X$ (or lower ordered interval transversal) iff: $\rho[x, y]=\rho[y, x]$ and if there is a lower (ordered interval) bisection function $d:[a, b] \times[a, b] \rightarrow[a, b]$ such that $(\mathrm{B})$.

A transversal lower ordered interval space is a set $X$ together with a given lower ordered interval transverse $\rho: X \times X \rightarrow[a, b]$ for $a \preccurlyeq b$ on $X$, where every increasing sequence $\left\{u_{n}\right\}_{n \in \mathbb{N}}$ of elements in $[a, b]$ has a unique element $u \in[a, b]$ as limit (in notation $u_{n} \rightarrow u$ or $\lim u_{n}=u$ ).

Let $\mathbb{S}$ be a nonempty totally ordered set. A mapping $N: \mathbb{S} \rightarrow[a, b]$ for $a \preccurlyeq b$ is called a lower function if it is increasing with inf $N=a$ and $\sup N=b$. We will denote by $\mathcal{L}$ the set of all lower functions.

Otherwise, a middle transversal ordered interval space is an upper and a lower transversal ordered interval space simultaneous.

A lower ordered statistical space is a pair $(X, \mathcal{F})$, where $X$ is an abstract set and $\mathcal{F}$ is a mapping of $X \times X$ into the set of all lower functions $\mathcal{L}$. We shall denote the lower function $\mathcal{F}(p, q)$ by $N_{p, q}(x)$ or $N_{p, q}$, whence the symbol $N_{p, q}(x)$ will denote the "value" of $N_{p, q}$ at $x \in \mathbb{S}$. The functions $N_{p, q}$ are assumed to satisfy the following conditions: $N_{p, q}=N_{q, p}, N_{p, q}(c)=a$ for some $c \in \mathbb{S}$, and

$$
N_{p, q}(x)=b \quad \text { for } \quad x \succ c \text { if and only if } p=q \text {, }
$$

and if $N_{p, q}(x)=b$ and $N_{q, r}(y)=b$ implies $N_{p, r}(\max \{x, y\})=b$ for all $p, q, r \in X$ and for all $x, y \in \mathbb{S}$.

In view of the condition $N_{p, q}(c)=a$ for some $c \in \mathbb{S}$, which evidently, implies that $N_{p, q}(x)=a$ for all $x \preccurlyeq c$. Thus the condition (Em) is equivalent to the statement: $p=q$ if and only if $N_{p, q}(x)=H(x)$, where $H(x)=a$ if $x \preccurlyeq c$ and $H(x)=b$ if $x \succ c$. 
An example of transversal lower ordered interval space is a lower ordered interval space which is a nonempty set $X$ together with the function $N_{p, q}(x)$ with the following properties: $N_{p, q}=N_{q, p}, N_{p, q}(c)=a$ for some $c \in \mathbb{S}$, (Em), and if there is a nondecreasing function $\tau:[a, b] \times[a, b] \rightarrow[a, b]$ with the property $\tau(t, t) \succcurlyeq t$ for all $t \in[a, b]$ such that

$(\mathrm{Nm})$

$$
N_{p, q}(\max \{x, y\}) \succcurlyeq \tau\left(N_{p, r}(x), N_{r, q}(y)\right)
$$

for all $p, q, r \in X$ and for all $x, y \succcurlyeq c$.

We notice, if we chosen a lower (ordered interval) bisection function $d:[a, b] \times$ $[a, b] \rightarrow[a, b]$ such that $d=\tau$ (from $(\mathrm{Nm}))$, then we immediate obtain that every lower ordered interval space, for $\rho[p, q]=N_{p, q}$ is a transversal lower ordered interval space; because in this case from $(\mathrm{Nm})$ for $y=x$ the following inequalities hold:

$$
\begin{array}{r}
\rho[p, q]=N_{p, q}(\max \{x, y\}) \succcurlyeq \tau\left(N_{p, r}(x), N_{r, q}(y)\right):= \\
:=d(\rho[p, r], \rho[r, q]) \succcurlyeq \inf \{\rho[p, r], \rho[r, q], d(\rho[p, r], \rho[r, q])\} .
\end{array}
$$

In connection with the preceding, a transversal lower ordered interval $T$-space is a pair $(X, \rho)$, where $X$ is a transversal lower ordered interval space and where the lower ordered transverse $\rho[u, v]=N_{u, v}(x)$ satisfying: $N_{u, v}=N_{v, u}, N_{u, v}(c)=a$ for some $c \in \mathbb{S}$ and $($ Em).

This space is a very characteristic example of transversal lower ordered interval spaces for further work.

The concept of a neighborhood in a lower transversal ordered interval space $X$ for the lower interval ordered transverse $\rho[p, q]=N_{p, q}(x)$ in $[a, b]$ for $a \preccurlyeq b$ is the following. If $p \in X, \mu \succ c$ for some $c \in \mathbb{S}$, and $\sigma \preccurlyeq b$, then an $(\mu, \sigma)$-neighborhood of $p$ denoted by $\mathcal{O}_{p}(\mu, \sigma)$, is defined by

$$
\mathcal{O}_{p}(\mu, \sigma)=\left\{q \in X: \rho[p, q]=N_{p, q}(\mu) \succ \sigma\right\} .
$$

Lemma 3. Let $(X, \rho)$ be a transversal lower ordered interval $T$-space, where the upper transverse $\rho[p, q]=N_{p, q}(x)$. If $\varepsilon_{1} \preccurlyeq \varepsilon_{2}$ and $r_{1} \preccurlyeq r_{2}$, then $\mathcal{O}_{p}\left(\varepsilon_{1}, r_{1}\right) \subset \mathcal{O}_{p}\left(\varepsilon_{2}, r_{2}\right)$.

Proof. Suppose $q \in \mathcal{O}_{p}\left(\varepsilon_{1}, r_{1}\right)$ so that $N_{p, q}\left(\varepsilon_{1}\right) \succcurlyeq r_{1}$. Then $N_{p, q}\left(\varepsilon_{2}\right) \succcurlyeq$ $N_{p, q}\left(\varepsilon_{1}\right) \succ r_{1} \succcurlyeq r_{2}$, whence, by definition, $q \in \mathcal{O}_{p}\left(\varepsilon_{2}, r_{2}\right)$. The proof is complete.

From the preceding facts, in this topology a sequence $\left\{p_{n}\right\}_{n \in \mathbb{N}}$ in $X$ converges to a point $p \in X$ (in notation $p_{n} \rightarrow p$ ) if for some $c \in \mathbb{S}$ and for every $\mu \succ c$ and every $\sigma \preccurlyeq b$, there exists an integer $\mathcal{M}(\mu, \sigma)$, such that $p_{n} \in \mathcal{O}_{p}(\mu, \sigma)$, i.e., $\rho\left[p, p_{n}\right]=N_{p, p_{n}}(\mu) \succ \sigma$ whenever $n \geq \mathcal{M}(\mu, \sigma)$.

Lemma 4. Let $(X, \rho)$ be a transversal lower ordered interval T-space, where the lower $\rho[u, v]=N_{u, v}(x)$. If $p_{n} \rightarrow p$, then $N_{p, p_{n}} \rightarrow N_{p, p}=H$, i.e., for some $c \in \mathbb{S}$ and every $x \succ c, N_{p, p_{n}}(x) \rightarrow N_{p, p}(x)=H(x)$, and conversely.

Proof. If $x \succ c$, the for every $\varepsilon \preccurlyeq b$ there exists an integer $\mathcal{M}(x, \varepsilon)$ such that $N_{p, p_{n}}(x) \succ \varepsilon$ whenever $n \geq \mathcal{M}(x, \varepsilon)$. But this means that 
$\lim _{n \rightarrow \infty} N_{p, p_{n}}(x)=b=N_{p, p}$. If $x=c$, then for every $n, N_{p, p_{n}}(c)=a$ and hence $\lim _{n \rightarrow \infty} N_{p, p_{n}}(c)=a=N_{p, p}(c)$. The converse is immediate. The proof is complete.

In further, if $T: X \rightarrow X$, then a function $u \mapsto N_{u, T u}(x)$ is ordered upper semicontinuous at $\xi \in X$ if $\left\{u_{n}\right\}_{n \in \mathbb{N}}$ is a sequence in $X$ and $u_{n} \rightarrow \xi$ $(n \rightarrow \infty)$ implies that $N_{\xi, T \xi}(x) \succcurlyeq \lim _{n \rightarrow \infty} N_{u_{n}, T u_{n}}(x)$ for every $x \in \mathbb{S}$.

In connection with the preceding, the sequence $\left\{p_{n}\right\}_{n \in \mathbb{N}}$ will be called fundamental in $X$ if for some $c \in \mathbb{S}$ and for each $\mu \succ c, \sigma \preccurlyeq b$ there is an integer $\mathcal{M}(\mu, \sigma)$ such that $\rho\left[p_{n}, p_{m}\right]=N_{p_{n}, p_{m}}(\mu) \succ \sigma$ whenever $n, m \geq$ $\mathcal{M}(\mu, \sigma)$ or in an equivalent entry as

$$
\lim _{n \rightarrow \infty}\left(\operatorname{diam}\left\{p_{k}: k \geq n\right\}\right)=b .
$$

In analogy with the completion concept of metric space, a transversal lower ordered interval space $X$ will be called complete if each fundamental sequence in $X$ converges to an element in $X$.

In this paper, a mapping $T$ of a transversal lower ordered interval space $(X, \rho)$ into itself, for $\rho[u, v]=N_{u, v}(x)$, will be called a lower ordered interval contraction if there exists an increasing function $\varphi: \mathbb{S} \rightarrow \mathbb{S}$ such that $t \preccurlyeq \varphi(t)$ for every $t \in \mathbb{S}$, for some $c \in \mathbb{S}$ that is

$$
\lim _{n \rightarrow \infty} N_{u, v}\left(\varphi^{n}(t)\right)=b \quad \text { for every } \quad t \succ c,
$$

and

$$
\begin{gathered}
N_{T u, T v}(x) \succcurlyeq \\
\succcurlyeq \inf \left\{N_{u, v}(\varphi(x)), N_{u, T u}(\varphi(x)), N_{v, T v}(\varphi(x)), N_{u, T v}(\varphi(x)), N_{v, T u}(\varphi(x))\right\}
\end{gathered}
$$

for all $u, v \in X$ and for all $x \succ c$.

Theorem 4. Let $(X, \rho)$ be a complete transversal lower ordered interval $T$-space, where the lower transverse $\rho[u, v]=N_{u, v}(x)$ is ordered upper semicontinuous and the lower bisection function $d:[a, b] \times[a, b] \rightarrow[a, b]$ is nondecreasing such that $d(t, t) \succcurlyeq t$ for all $t \in[a, b]$. If $T$ is any lower ordered interval contraction mapping of $X$ into itself, then there is a unique point $p \in X$ such that $T p=p$. Moreover, $T^{n} q \rightarrow p$ for each $q \in X$.

The proof of this statement is a totally analogous with the preceding proof of Theorem 1. Also a totally analogous with Theorem 2 is the following statement. We notice that with this result are connection: B o c şan-Const antin [7], Constantin-Istrăteşc u [12], Górn i ewicz [20], Istrăt eş cu [24], $\mathrm{K}$ a l e va-S eik kal a [25], O n i c e s c u [36], S chweize r- S c lar [43], S eh g a l [44], S e h g a l et. al. [45], S h e r w o o d [46], Š er s t n e v [47], S h a r m a [48], and Singh et. al. [49]. 
Theorem 5. Let $(X, \rho)$ be a complete transversal ordered lower interval $T$-space, where the upper transverse $\rho[u, v]=N_{u, v}(x)$ is ordered upper semicontinuous and the lower bisection function $d:[a, b] \times[a, b] \rightarrow[a, b]$ is nondecreasing such that $d(t, t) \succcurlyeq t$ for all $t \in[a, b]$. If there exists an increasing function $\varphi: \mathbb{S} \rightarrow \mathbb{S}$ such that $t \preccurlyeq \varphi(t)$ for every $t \in \mathbb{S}$ with the property (Ar) for some $c \in \mathbb{S}$ and if for each $u \in X$ there is a positive integer $n=n(u)$ such that

$$
\begin{gathered}
N_{T^{n}(u), T^{n}(v)}(x) \succcurlyeq \\
\succcurlyeq \inf \left\{N_{u, v}(\varphi(x)), N_{u, T^{n} u}(\varphi(x)), N_{v, T^{n} v}(\varphi(x)), N_{u, T^{n} v}(\varphi(x)), N_{v, T^{n} u}(\varphi(x))\right\}
\end{gathered}
$$

for every $v \in X$ for every $x \succ c$, then $T$ has exactly one fixed point $p \in X$ and $T^{n} q \rightarrow p$ for every $q \in X$.

A brief proof of this statement based on the preceding facts, is special case for transversal lower probabilistic $T$-space, may be found in Tasković [55].

As immediate consequence of the preceding Theorem 4 we obtain directly the following interesting cases of lower ordered interval contractive mappings:

(M') There exists an increasing function $\varphi: \mathbb{S} \rightarrow \mathbb{S}$ with the property (Ar) for some $c \in \mathbb{S}$ such that for each $u, v \in X$ and for every $x \succ c$ the following inequality holds

$$
N_{T u, T v}(x) \succcurlyeq N_{u, v}(\varphi(x)) .
$$

If the interval $[a, b]$ for $a \preccurlyeq b$ is a totally ordered set, then as a directly consequence of Theorem 4 we obtain the following case of lower ordered interval contractive mappings:

$\left(\mathrm{N}^{\prime}\right)$ There is an increasing function $\varphi: \mathbb{S} \rightarrow \mathbb{S}$ such that $t \preccurlyeq \varphi(t)$ for every $t \in \mathbb{S}$ with the property (Ar) and for all $u, v \in X$ and for every $x \succ c$ that is

$$
\begin{gathered}
N_{T u, T v}(x) \succcurlyeq \\
\succcurlyeq \min \left\{N_{u, v}(\varphi(x)), N_{u, T u}(\varphi(x)), N_{v, T v}(\varphi(x)), N_{u, T v}(\varphi(x)), N_{v, T u}(\varphi(x))\right\} .
\end{gathered}
$$

If $[a, b] \subset \mathbb{R}_{+}^{0}$ for $a \preccurlyeq b$ and $\mathbb{S}=\mathbb{R}$, then we have the following two cases of lower ordered interval contractive mappings:

(S') There exists a constant $0<k<1$ such that for each $p, q \in X$, for some $c \in \mathbb{R}$ and for every $x>c$ the following inequality holds

$$
N_{T p, T q}(x) \geq N_{p, q}\left(\frac{x}{k}\right) .
$$

(R') There exists a constant $0<k<1$ such that for each $u, v \in X$, for some $c \in \mathbb{R}$ and for every $x>c$ the following inequality holds

$$
N_{T u, T v}(k x) \geq \min \left\{N_{u, v}(x), N_{u, T u}(x), N_{v, T v}(x), N_{u, T v}(x), N_{v, T u}(x)\right\} .
$$

Annotations. In connection with the preceding facts, we notice that third characteristic example of a transversal lower ordered interval space is so-called lower edges ordered space of the form $(X, \rho)$, where $X$ is a nonempty set and $\rho: X \times X \rightarrow(a, b]$ for $a \preccurlyeq b$ is given lower edges ordered transverse with the 
properties: $\rho[x, y]=b$ if and only if $x=y, \rho[x, y]=\rho[y, x]$, and if there is a function $d:(a, b]^{2} \rightarrow(a, b]$ such that

$$
\inf \{\rho[x, z], \rho[z, y], d(\rho[x, z], \rho[z, y])\} \preccurlyeq \rho[x, y]
$$

for all $x, y, z \in X$. In this sense, an example, we can suppose that every increasing sequence of elements in $(a, b]$ has a unique element as limit in $(a, b]$.

In this sense, in lower edges ordered space $(X, \rho)$, the sequence $\left\{x_{n}\right\}_{n \in \mathbb{N}}$ lower converges (or lower edges ordered converges) to a point $x \in X$ (in notation $x_{n} \rightarrow x$ $(n \rightarrow \infty))$ if for some increasing sequence $\left\{b_{n}\right\}_{n \in \mathbb{N}}$ in $(a, b]$ which converges to $b$ the following inequality holds in the form

$$
\rho\left[x, x_{n}\right] \succ b_{n} \text { for all } n \in \mathbb{N},
$$

or for $n$ and $m$ large enough. On the other hand, in connection with this, the sequence $\left\{x_{n}\right\}_{n \in \mathbb{N}}$ in $X$ will be called lower fundamental (or lower edges ordered fundamental) if the following inequality holds in the form

$$
\rho\left[x_{n}, x_{m}\right] \succ b_{n} \quad \text { for all } n, m \in \mathbb{N} \quad(n<m),
$$

or for $n$ and $m$ large enough, where the increasing sequence $\left\{b_{n}\right\}_{n \in \mathbb{N}}$ in $(a, b]$ converges to $b$.

An lower edges ordered space $(X, \rho)$ is called lower complete (or lower edges ordered complete) if any lower fundamental sequence $\left\{x_{n}\right\}_{n \in \mathbb{N}}$ in $X$ is lower convergent (to a point of $X$, of course). For further results on lower edges ordered spaces see: Ta s k ovi ć [57].

Coincidence on middle transversal ordered interval spaces. In conection with this, the mappings $T, f: X \rightarrow X$ of a transversal middle ordered interval space $X:=(X, \rho, r)$ into itself for $\rho[u, v]=M_{u, v}(x)$ and $r[u, v]=N_{u, v}(x)$, will be called middle ordered interval contractions if there exists an increasing function $\varphi: \mathbb{S} \rightarrow \mathbb{S}$ such that $t \preccurlyeq \varphi(t)$ for every $c \in \mathbb{S}$, and for some $x \in \mathbb{S}$ that is (As) and (Ar) with the properties

$$
\begin{gathered}
M_{T u, T v}(x) \preccurlyeq \\
\preccurlyeq \sup \left\{M_{f u, f v}(\varphi(x)), M_{f u, T u}(\varphi(x)), M_{f u, T v}(\varphi(x)), M_{f v, T v}(\varphi(x)), M_{f v, T u}(\varphi(x))\right\}
\end{gathered}
$$

and

$$
\begin{gathered}
N_{T u, T v}(x) \succcurlyeq \\
\succcurlyeq \inf \left\{N_{f u, f v}(\varphi(x)), N_{f u, T u}(\varphi(x)), N_{f u, T v}(\varphi(x)), N_{f v, T v}(\varphi(x)), N_{f v, T u}(\varphi(x))\right\}
\end{gathered}
$$

for all $u, v \in X$ and for every $x \succ c$.

In conection with this we notice a middle transversal ordered interval space $X$ will be called middle complete if $X$ is upper and lower complete, simultaneous.

Now, from Theorems 1 and 4 directly we obtain the following result for middle transversal ordered interval spaces in the following form as. 
Theorem 6. Let $(X, \rho, r)$ be a middle complete middle transversal ordered interval $T, f$-space where $\rho[u, v]=M_{u, v}(x)$ is ordered lower semicontinuous and $r[u, v]=N_{u, v}(x)$ is ordered upper semicontinuous. Let the upper bisection function $g$ be a nondecreasing such that $g(t, t) \preccurlyeq t$ and the lower bisection function $d$ be a nondecreasing such that $d(t, t) \succcurlyeq t$ for every $t \in[a, b]$ If the mappings $T, f: X \rightarrow X$ are any middle ordered interval contractions mappings of $X$ into itself, if for an arbitrary fixed $x_{0} \in X$ there exists $x_{1} \in X$ such that $T\left(x_{0}\right)=f\left(x_{1}\right)$, and if $T$ and $f$ commutes, then $T$ and $f$ have a common unique fixed point $\xi \in X$, i.e., $\xi=T(\xi)=f(\xi)$ for some $\xi \in X$.

The proof of this statement is a completely analogy with the proofs of the preceding statements for upper and lower transversal ordered interval spaces.

\section{Applications and Consequences}

We notice that numerous questions in physics, chemistry, biology, and economics lead to nonlinear problems. In this sense the preceding concept of transversal ordered (upper and lower) interval spaces can be have fundamental role. An example of this is nonlinear numerical analysis by L. Collatz [11]. Also, this concept have very important applications in quantum particle physics particulary in connection with both string and $\varepsilon^{\infty}$-theory which were given and studied by El Naschie [18].

In connection with this, as a consequence of the preceding facts, we have the following applications.

The functions $\rho, r: X \times X \rightarrow[a, b]$ for $a \preccurlyeq b$ are called middle ordered interval transverses on $X$ (or middle ordered interval transversals) iff: $\rho[x, y]=\rho[y, x], r[x, y]=r[y, x]$ and if there is a middle (ordered interval) bisection function $\psi:[a, b] \times[a, b] \rightarrow[a, b]$ such that (A) with $\rho$ and (B) with $r$.

A transversal middle ordered interval space is a set $X:=(X, \rho, r)$ together with given middle ordered interval transverses $\rho, r: X \times X \rightarrow[a, b]$ for $a \preccurlyeq b$ on $X$, where every increasing (or decreasing) sequence $\left\{u_{n}\right\}_{n \in \mathbb{N}}$ of elements in $[a, b]$ has a unique element $u \in[a, b]$ as limit (in notation $u_{n} \rightarrow u$ or $\lim u_{n}=u$ ).

We notice that any metric space is a transversal middle ordered interval space, i.e., an upper and lower transversal ordered interval space, simultaneous.

K. M e n g e r introduced in 1928 and 1942 the notion of probabilistic metric spaces. O. K a l e va and S. S e i k ka la proved in 1984 that each Menger space, which is a special probabilistic metric space, can be considered as a fuzzy metric space.

Interesting, every fuzzy metric space is, de facto, also a lower ordered transversal space.

Let $X$ be a nonempty set, $E$ a set of all upper semicontinuous normal convex fuzzy numbers, $G$ a set of all nonnegative fuzzy numbers of $E$ and $m: X \times X \rightarrow G$. 
A quadruple $(X, m, L, R)$ is called a fuzzy metric space with $m$ as a fuzzy metric if $L, R:[0,1]^{2} \rightarrow[0,1]$ are symmetric functions, nondecreasing in both arguments, $L(0,0)=0$ and $R(1,1)=1$ such that $m(x, y)=0$ iff $x=y, m(x, y)=$ $m(y, x)$,

$$
m(x, y)_{(s+r)} \leq R\left(m(x, z)_{(s)}, m(z, y)_{(r)}\right)
$$

for all $x, y, z \in X$, where $s \geq \lambda_{1}(x, z), r \geq \lambda_{1}(z, y)$ and $s+r \geq \lambda_{1}(x, y)$, and such that

$$
m(x, y)_{(s+u)} \geq L\left(m(x, z)_{(s)}, m(z, y)_{(u)}\right)
$$

for all $x, y, z \in X$, where $s \leq \lambda_{1}(x, z), u \leq \lambda_{1}(z, y)$, and $s+u \leq \lambda_{1}(x, y)$.

In connection with this, let $P:=[0,1]$ and we chosen a lower bisection function $d:[0,1]^{2} \rightarrow[0,1]$ such that $d=L$ (with the preceding properties), then we immediately obtain that every fuzzy metric space is a lower ordered transversal space. On the other hand, if for the upper bisection function $g:[0,1]^{2} \rightarrow[0,1]$ we chosen that is $g=R$ (with the preceding properties), then we have that every fuzzy metric space is an upper ordered transversal space. This means, common with the preceding, that every fuzzy metric space, de facto, is an ordered middle transversal space.

Following At a n as sov [4] and [5], also, G e or g e - Ve e r a m a n i [19], J. H. P a r k [39] have defined intuitionistic fuzzy metric spaces and obtained several classical statements on this structure. A la c a et. al. [2] and [3] studied new properties of intuitionistic fuzzy metric spaces.

In this sense, a 5-tuple $(X, M, N, *, \diamond)$ is said to be an intuitionistic fuzzy metric space if $X$ is an arbitrary set, $*$ is a continuous $t$-norm, $\diamond$ is a continuous $t$-conorm and $M, N$ are fuzzy sets on $X \times X \times \mathbb{R}_{+}\left(\mathbb{R}_{+}:=(0, \infty)\right)$ satisfying, for all $x, y, z \in X$ and $s, t \in \mathbb{R}_{+}$, the following conditions: $M(x, y, t)+N(x, y, t) \leq 1$, $M(x, y, t)>0, M(x, y, t)=1$ if and only if $x=y, M(x, y, t)=M(y, x, t)$, $M(x, y, t) * M(y, z, s) \leq M(x, z, t+s), t \mapsto M(x, y, t)$ and $t \mapsto N(x, y, t)$ are continuous, $N(x, y, t)>0, N(x, y, t)=N(y, x, t), N(x, y, t)=0$ if and only if $x=y$, and $N(x, y, t) \diamond N(y, z, s) \geq N(x, z, t+s)$.

Then $(M, N)$ is called an intuitionistic fuzzy metric on $X$. The functions $M(x, y, t)$ and $N(x, y, t)$ denote the degree of nearness and the degree of nonnearness between $x$ and $y$ with respect to $t$, respectively.

In an intuitionistic fuzzy metric space $X$, we notice that $t \mapsto M(x, y, t)$ is nondecreasing, and $t \mapsto N(x, y, t)$ is nonincreasing for all $x, y \in X$.

Every fuzzy metric space $(X, M, *)$ is an intuitionistic fuzzy metric space of the form $(X, M, 1-M, *, \diamond)$ such that $t$-norm $*$ and $t$-conorm $\diamond$ are associated, i.e., $x \triangleright y:=1-((1-x) *(1-y))$ for any $x, y \in X$.

We notice that, de facto, as an important example of transversal middle ordered interval spaces we have an intuitionistic metric space.

Indeed, if for every lower ordered bisection function $d:[a, b] \times[a, b] \rightarrow[a, b]$ for $a \preccurlyeq b,[a, b]:=[0,1]$, we chosen that is $d:=M(x, y, t) * M(y, z, s)$, and if for upper ordered bisection function $g:[a, b] \times[a, b] \rightarrow[a, b]$ for $[a, b]:=[0,1]$ we chosen that is $g:=N(x, y, t) \diamond N(y, z, s)$, then we have, directly, that every intuitionistic metric space is a transversal middle ordered interval space.

For recently result of fixed points on intuitionistic metric spaces see: $\mathrm{P}$ a $\mathrm{r} \mathrm{k}$ [39], $\mathrm{S}$ a a d a t i et al. [41], Sharma [48], S ing h et al. [49], Moh a m a d [33], 
Pant [38], Kramosil-M i chalek [32], R a z ani [40], S a d a t i [42], and others.

On the other hand, S a a d a t i et al. [41] have defined the concept of $\mathcal{L}$-fuzzy metric spaces and proved a common fixed point theorem for a pair of commutative mappings with linear contractive conditions. The definition of $\mathcal{L}$-fuzzy metric spaces is given by $\mathrm{S}$ a a d a t i et al. [41] in the following sense.

Let $\mathcal{L}:=(L, \preccurlyeq)$ be a complete lattice, and $U$ a nonempty set called universe. An $\mathcal{L}$-fuzzy set $A$ on $U$ is defined as a mapping $A: U \rightarrow L$. For each $u \in U$, $A(u)$ represents the degree (in $L$ ) to which $u$ satisfies $A$. We define $0:=\inf L$ and $1:=\sup L$.

A triangular norm $(t$-norm) on $\mathcal{L}$ is a mapping $\mathcal{L}: L \times L \rightarrow L$ satisfying for all $x, y, z, x_{1}, y_{1} \in L$ the following conditions: $\mathcal{L}(x, 1)=x, \mathcal{L}(x, y)=\mathcal{L}(y, x)$, $\mathcal{L}(x, \mathcal{L}(y, z))=\mathcal{L}(\mathcal{L}(x, y), z)$, and $x \preccurlyeq x_{1}$ and $y \preccurlyeq y_{1}$ implies $\mathcal{L}(x, y) \preccurlyeq \mathcal{L}\left(x_{1}, y_{1}\right)$.

The 3 -tuple $(X, M, \mathcal{L})$ is said to be an $\mathcal{L}$-fuzzy metric space if $X$ is an arbitrary nonempty set, $\mathcal{L}$ is a continuous $t$-norm on $\mathcal{L}$ and $M$ is an $\mathcal{L}$-fuzzy set on $X \times X \times \mathbb{R}_{+}$ satisfying for all $x, y, z \in X$ and $t, s \in \mathbb{R}_{+}$the following conditions: $M(x, y, t)>0$, $M(x, y, t)=1$ for every $t \in \mathbb{R}_{+}$if and only if $x=y, M(x, y, t)=M(y, x, t)$, $\mathcal{L}(M(x, y, t), M(y, z, s)) \preccurlyeq M(x, z, t+s)$, and $t \mapsto M(x, y, t)$ is continuous. In this case $M$ is called an $\mathcal{L}$-fuzzy metric.

In this sense, if for lower ordered bisection function $d:[a, b] \times[a, b] \rightarrow[a, b]$ for $a \preccurlyeq b,[a, b]=L$, we chosen that is $d=\mathcal{L}: L \times L \rightarrow L$ (with the preceding properties) and $\rho[x, y]:=M(x, y, t)$, then we have, directly, that every $\mathcal{L}$-fuzzy metric space is a transversal lower ordered interval space. This means, common with the preceding, that every $\mathcal{L}$-fuzzy metric space, de facto, is a transversal lower ordered space.

Also, in the connection with the preceding transversal edges spaces, the functions $\rho, r: X \times X \rightarrow[a, b]$ for $a \preccurlyeq b$ are called middle edges ordered transverses on $X$ (or middle edges ordered transversals) iff: $\rho$ is an upper and $r$ is a lower edges ordered transverse simultaneously.

A middle edges ordered space is a set $X:=(X, \rho, r)$ together with given middle edges ordered transverses $\rho, r: X \times X \rightarrow[a, b]$ for $a \preccurlyeq b$ on $X$. We notice that any intuitionistic fuzzy metric space is a middle edges ordered space, i.e., an upper and lower edges ordered space, simultaneously. For further facts of this see: Tasković [57].

Also, we notice that any $\mathcal{L}$-fuzzy metric space is an example of lower edges ordered spaces.

Asymptotic conditions on spring ordered spaces. Let $X$ be a nonempty set and let $P:=(P, \preccurlyeq)$ be a partially ordered set. The function $A: X \times X \rightarrow$ $[a, b) \subset P$ for $a \preccurlyeq b$ is called an upper spring ordered transverse on a nonempty set (or upper spring ordered transversal) iff $A(x, y)=a$ if and only if $x=y$ for all $x, y \in X$.

An upper spring ordered transversal space $X:=(X, A)$ is a nonempty set $X$ together with a given upper spring ordered transverse $A$ on $X$, where every decreasing sequence $\left\{u_{n}\right\}_{n \in \mathbb{N}}$ of elements in $[a, b)$ has a unique element $u \in[a, b)$ as limit (in notation $u_{n} \rightarrow u(n \rightarrow \infty)$ ). 
In 1986 we investigated the concept of upper spring ordered TCS-convergence in a space $X$, i.e., and upper spring ordered transversal space $X:=(X, A)$ satisfies the condition of upper spring ordered TCS-convergence iff $x \in X$ and if $A\left(T^{n}(x), T^{n+1}(x)\right) \rightarrow a(n \rightarrow \infty)$ implies that $\left\{T^{n}(x)\right\}_{n \in \mathbb{N}}$ has a convergent subsequence in $X$, by Tasković [57].

We notice that the sequence $\left\{x_{n}\right\}_{n \in \mathbb{N}}$ in the upper spring ordered transversal space $X:=(X, A)$ is convergent in notation $x_{n} \rightarrow x(n \rightarrow \infty)$ iff $A\left(x_{n}, x\right) \rightarrow a$ as $n \rightarrow \infty$.

Theorem 7. Let $T$ be a mapping of upper spring ordered transversal space $X:=$ $(X, A)$ into itself, where $X$ satisfies the condition of upper spring ordered TCSconvergence. Suppose that for all $x, y \in X$ there exist a sequence of functions $\left\{\alpha_{n}(x, y)\right\}_{n \in \mathbb{N}}$ such that $\alpha_{n}(x, y) \rightarrow a(n \rightarrow \infty)$ and positive integer $m(x, y)$ such that

$$
A\left(T^{n}(x), T^{n}(y)\right) \preccurlyeq \alpha_{n}(x, y) \quad \text { for all } n \geq m(x, y),
$$

where $x \mapsto A(x, T(x))$ is ordered lower semicontinuous, then $T$ has a unique fixed point $\xi \in X$ and $T^{n}(x) \rightarrow \xi(n \rightarrow \infty)$ for each $x \in X$.

A proof of this statement may be found in: Tas k ovi ć [57]. For second proof of this statement see: Ta s k o vi ć [51].

We notice that, from the preceding facts of this paper, we can give the following local form of this statement.

Theorem 8. (Localization of $(\mathrm{R})$ ). Let $T$ be a mapping of upper spring ordered transversal space $X:=(X, A)$ into itself, where $X$ satisfies the condition of upper spring ordered TCS-convergence. Suppose that for each $x \in X$ there exist a sequence of functions $\left\{\alpha_{n}(x, T(x))\right\}_{n \in \mathbb{N}}$ such that $\alpha_{n}(x, T(x)) \rightarrow a(n \rightarrow \infty)$ and positive integer $m(x, T(x))$ such that

$$
A\left(T^{n}(x), T^{n+1}(x)\right) \preccurlyeq \alpha_{n}(x, T(x)) \text { for all } n \geq m(x, T(x)),
$$

where $x \mapsto A(x, T(x))$ is ordered lower semicountinuous, then $T$ has at least one fixed point in $X$.

In the next, let $X$ be a nonempty set and let $P:=(P, \preccurlyeq)$ be a partially ordered set. The function $A: X \times X \rightarrow(a, b] \subset P$ for $a \preccurlyeq b$ is called a lower spring ordered transverse on a nonempty set (or lower spring ordered transversal) iff $A(x, y)=b$ if and only if $x=y$ for all $x, y \in X$.

A lower spring ordered transversal space $X:=(X, A)$ is a nonempty set $X$ together with a given lower spring ordered transverse $A$ on $X$, where every increasing sequence $\left\{u_{n}\right\}_{n \in \mathbb{N}}$ of elements in $(a, b]$ has a unique element $u \in(a, b]$ as limit (in notation $u_{n} \rightarrow u(n \rightarrow \infty)$ ).

In 1986 we investigated the concept of lower spring ordered TCS-convergence in a space $X$, i.e., a lower spring ordered transversal space $X:=(X, A)$ satisfies the condition of lower spring ordered TCS-convergence iff $x \in X$ and if $A\left(T^{n}(x), T^{n+1}(x)\right) \rightarrow b(n \rightarrow \infty)$ implies that $\left\{T^{n}(x)\right\}_{n \in \mathbb{N}}$ has a convergent subsequence in $X$ by Tasković [57].

We notice that the sequence $\left\{x_{n}\right\}_{n \in \mathbb{N}}$ in the lower spring ordered transversal space $X:=(X, A)$ is convergent in notation $x_{n} \rightarrow x(n \rightarrow \infty)$ iff $A\left(x_{n}, x\right) \rightarrow b$ as $n \rightarrow \infty$. 
Theorem 9. Let $T$ be a mapping of lower spring ordered transversal space $X:=$ $(X, A)$ into itself, where $X$ satisfies the condition of lower spring ordered TCSconvergence. Suppose that for all $x, y \in X$ there exist a sequence of functions $\left\{\alpha_{n}(x, y)\right\}_{n \in \mathbb{N}}$ such that $\alpha_{n}(x, y) \rightarrow b(n \rightarrow \infty)$ and positive integer $m(x, y)$ such that

$$
A\left(T^{n}(x), T^{n}(y)\right) \succcurlyeq \alpha_{n}(x, y) \quad \text { for all } n \geq m(x, y),
$$

where $x \mapsto A(x, T(x))$ is ordered upper semicontinuous, then $T$ has a unique fixed point $\xi \in X$ and $T^{n}(x) \rightarrow \xi(n \rightarrow \infty)$ for each $x \in X$.

A proof of this statement may be found in: Tas k o vi ć [57]. For second proof of this statement see: Ta s k ović [51].

We notice that, from the preceding facts of this paper, we can give the following local form of this statement.

Theorem 10. (Localization of $(\mathrm{G})$ ). Let $T$ be a mapping of lower spring ordered transversal space $X:=(X, A)$ into itself, where $X$ satisfies the condition of lower spring ordered TCS-convergence. Suppose that for each $x \in X$ there exist a sequence of functions $\left\{\alpha_{n}(x, T(x))\right\}_{n \in \mathbb{N}}$ such that $\alpha_{n}(x, T(x)) \rightarrow b(n \rightarrow \infty)$ and positive integer $m(x, T(x))$ such that

$$
A\left(T^{n}(x), T^{n+1}(x)\right) \succcurlyeq \alpha_{n}(x, T(x)) \quad \text { for all } n \geq m(x, T(x)),
$$

where $x \mapsto A(x, T(x))$ is ordered upper semicountinuous, then $T$ has at least one fixed point in $X$.

We notice that a middle spring ordered transversal space $X$ is an upper spring ordered transversal space and a lower spring ordered transversal space simultaneous. For further facts on middle spring ordered transversal spaces see: Tas k ović [57].

\section{REFERENCES}

[1] A. Appert and Ky Fan, Espaces topologiques intermédiaires. Problème de la distanciation, Actuelités scient., Paris, 1951

[2] C. Alaca, D. Turkoglu, C. Yildiz, Fixed points in intuitionistic fuzzy metric spaces. Chaos, Solitons \& Fractals 2006; 29:1073-8.

[3] C. Alaca, H. Efe, C. Yildiz, On completion of intuitionistic fuzzy metric spaces. Chaos, Solitons \& Fractals (2006), doi:10.1016/j.chaos.2006.01.039.

[4] K. Atanassov, Intuitionistic fuzzy sets. In: Sgurev V, editor. VII ITKRs Session, Sofia, June 1983 (Central Sci. and Techn. Library, Bulg. Academy of Sciences, 1984).

[5] K. Atanassov, Intuitionistic fuzzy sets. Fuzzy Sets Syst 1986; 20:87-96.

[6] P. Azzimondi and C. Scaravelli, Un teorema del punto unito in spazi metrici generalizzati, Riv. Mat. Univ. Parma, 5 (1979), 773-780.

[7] G. Bocşan and G. Constantin, The Kuratowski function and some applications to probabilistic metric spaces, Atti dela Academia Nazionale dei Lincei, 8 (1973), 236240 .

[8] E.W. Chittenden, On the equivalence of ecart and voisinage, Trans. Amer. Math. Soc., 18 (1917), 161-166. 
[9] M. Cicchese, Distanze generalizzate uniformemente continue, Riv. Mat. Univ. Parma, 3 (1974), 133-137.

[10] J. Colmez, Espaces à écart généralisé régulier, C. R. Acad. Sci. Paris, 224 (1947), p.p. 372 .

[11] L. Collatz, Funktionalanalysis und numerische Mathematik. Grundld. math. Wiss. 120, Springer-Verlag, Berlin, Heidelberg, New York, 1968.

[12] G. Constantin and I. Istrăţescu, Elements of Probabilistic Analysis, Kluwer Academic Publishers, 1989.

[13] S. Czerwik, Nonlinear set-valued contraction mappings in b-metric spaces, Atti. Sem. Math. Fis. Univ. Modena, 46 (1998), 263-276.

[14] R. Doss, Sur la condition de régularité pour l'ecart abstrait. Écart abstrait symétrique et régulier, C. R. Acad. Sci. Paris, 223 (1946), 14-16, 1087-1088.

[15] Kurpelj, N. S. Projekciono iterativne metodii rešenia operatornih uravnenii, Kiev, 1968., 243 p.p.

[16] Kwapisz, M. Some generalization of an abstract contraction mapping principle, Nonlinear Anal. Theory, Meth. Appl., 3 (1979), 293-302.

[17] F. Cammaroto, and Lj. Kočinac, Some results on $\omega_{\mu}$-Metrizable and Related Spaces, Boll. U. M. I., 7-B (1993), 607-629.

[18] M.S. El Naschie, A review of E-infinity theory and the mass spectrum of high energy particle physics. Chaos, Solitons \& Fractals 2004;19:209-36.

[19] A. George, P. Veeramani, On some results in fuzzy metric spaces. Fuzzy Sets Syst 1994;64:395-9.

[20] L. Górniewicz, Topological fixed point theory of multivalued mappings, Kluwer Acad. Publ. Dordrecht, 1999.

[21] M. Fréchet, La notion d'écart et le calucl fonctionnel, C. R. Acad. Sci. Paris, 140 (1905), 772-774.

[22] M. Fréchet, Sur quelques points du calcul fonctionnel, Thèse, Paris, 1905. Rendiconti Circolo Mat. Palermo, 22 (1906), 1-74.

[23] A. H. Frink, Distance functions and the metrization problem, Bull. Amer. Math. Soc., 43 (1937), 133-142.

[24] V. Istrăţescu, Introducere in teoria spatiilor metrice probabilistic applicatti, Edifura Technica, Bucarest, 1974.

[25] O. Kaleva and S. Seikkala, On fuzzy metric spaces, Fuzzy Sets and Systems, 12 (1984), 215-229.

[26] L.V. Kantorovitch, The method of successive approximations for functional equations, Acta Math., 71 (1939), 63-97.

[27] Dj.R. Kurepa, Tableaux ramifiés d'ensambles. Espaces pseudo-distanciés, C. R. Acad. Paris, 198 (1934), 1563-1565. Ensembles ordonnés et ramifiés, Thèse, Paris, 1935, p.p. 1-138; Publ. Inst. Math. Belgrade, 4 (1935), 1-138.

[28] Dj.R. Kurepa, Sur les classes (E) et (D), Publ. Inst. Math. Belgrade, 55 (1936), 124-132.

[29] Dj.R. Kurepa, Un critère de distanciabilitè, Mathematica (Cluj), 13 (1937), 59-65. 
[30] Dj.R. Kurepa, Distanca numerica. Distanca non numerica, Publ. Seminario Matematica, Bari, 1963.

[31] Dj.R. Kurepa, On the existence of pseudometric non totally orderable spaces, Glasnik Mat. Fiz. Astr. Zagreb, 18 (1963), 183-194.

[32] J. Kramosil, J. Michalek, Fuzzy metric and statistical metric space, Kybernetika 1975;11:326-34.

[33] A. Mohamad, Fixed-point theorems in intuitionistic fuzzy metric space. Chaos, Solitons \& Fractals, Volume 34, Issue 5, December 2007, 1689-1695.

[34] K. Menger, Untersuchungen über allgemaine Metric, Math. Annalen, 100 (1928), 75-163.

[35] K. Menger, Statistical metrics, Proc. Nat. Acad. Sci., USA, 28 (1942), 535-537.

[36] O. Onicescu, Nombres et Systèmes Aléatoires, Éditions de L'Académie de la R. P. Roumaine, Bucarest, 1964.

[37] P. Papić, Sur une classe d'espaces abstrait, Glasnik Mat. Fiz. Astr. Zagreb, 9 (1954), 197-216.

[38] R.P. Pant, V. Pant, Common Fixed Points under Strict Contractive Conditions, Jour. Math. Anal. Appl. 2000;248:327-332.

[39] J.H. Park, Intuitionistic fuzzy metric spaces. Chaos, Solitons \& Fractals 2004;22:103946.

[40] A. Razani, Existence of fixed point for the nonexpansive mapping of intuitionistic fuzzy metric spaces. Chaos, Solitons \& Fractals 2006;30:367-373.

[41] R. Saadati, A. Razani, H. Adibi, A common fixed point theorem in $\mathcal{L}$-fuzzy metric spaces. Chaos, Solitons \& Fractals (2006), doi:10.1016/ j.chaos.2006.01.023.

[42] R. Saadati, Notes to the paper "Fixed points on intuitionistic fuzzy metric spaces" and its generalization to $\mathcal{L}$-fuzzy metric spaces. Chaos, Solitons \& Fractals (2006), doi:10.1016/j.chaos.2006.05.005.

[43] B. Schweizer and A. Sklar, Statistical metric spaces, Pacific J. Math., 10 (1960), 313-334.

[44] V.M. Sehgal, Some fixed point theorems in functional analysis and probability, Ph. D. dissertation, Wayne State Univ., 1966.

[45] V.M. Seghal and A.T. Bharucha-Reid, Fixed points of contraction Mappings on Probabilistic Metric Spaces, Math. Systems Theory, 6 (1972), 97-102.

[46] H. Sherwood, Complete Prbabilistic Metric Spaces, Z. Wahrscheinli-chkeitstheorie verw. Geb., 20 (1971), 117-128.

[47] A.N. Šerstnev, On the probabilistic generalization of metric spaces, Kazan. gosudarst. Univ., učenye Zapiski, 124 (1967), 109-119.

[48] S. Sharma, Common fixed point in fuzzy metric spaces. Fuzzy Sets and Systems 2002;127:345-52.

[49] B. Singh, M.S. Chauhan, Common fixed points of compatible maps in fuzzy metric spaces. Fuzzy Sets and Systems 2000;115:471-5.

[50] R. Sikorski, Remark on some topological spaces of high power, Fund. Math. 37 (1950), 128-136. 
[51] M.R. Tasković, On the convergence of certain sequences and some applications - II, Publ. Inst. Math. (Beograd), 22(1977), 271-281.

[52] M.R. Tasković, Some new principles in fixed point theory, Math. Japonica, 35 (1990), 645-666.

[53] M.R. Tasković, Nonlinear Functional Analysis, Fundamental elements of theory, First Book. Monographs, Zavod za udžbenike i nastavna sredstva, Beograd 1993, 812 p.p. (Serbo-Croatian). English summary, Comments only new main results of this book. Vol. 1 (1993), 713-752.

[54] M.R. Tasković, Transversal spaces, Math. Moravica, 2 (1998), 133-142.

[55] M.R. Tasković, Fixed points on transversal probabilistic spaces, Math. Moravica, 3 (1999), 77-82.

[56] M.R. Tasković, Transversal intervally spaces, Math. Moravica, 7 (2003), 91-106.

[57] M.R. Tasković, Theory of transversal point, spaces and forks, Monographs of a new mathematical theory, VIZ-Beograd, 2005 (in Serbian), 1054 pages. English summary: 1001-1022.

[58] M.R. Tasković, Some results in the fixed point theory, Pub. Inst. Math. (Beograd), 20(1976), 231-242.

[59] S. Todorčević, On a theorem of Dj. Kurepa, in: Topology and order structure I, Math. Centre Tracts 142, Amsterdam, 1981, 173-176.

[60] A. Wald, On a statistical generalization of metric spaces, Proc. Nat. Acad. Sci. U.S.A., 29 (1943), 196-197.

[61] T. Wažewski, Sur un procédé de prouver la convergence des approximations succesive sans ultisations des séries comparaison, Bull. Acad. Polon. Sci. Math. Astronom. Phys., B (1960), 47-52.

\author{
Milan R. TASKović \\ Faculty of Mathematics \\ 11000 Belgrade, P.O. Box 550 \\ Serbia \\ Home Address: \\ Milan R. TAsković \\ Nehruova 236 \\ 11070 BELGRADE \\ SERBIA \\ E-mail address: andreja@predrag.us
}

